\title{
The type II antitoxin HigA is a key virulence regulator in Pseudomonas aeruginosa
}

Yingjie Song, ${ }^{1 \#}$ Siping Zhang, ${ }^{2 \#}$ Guihua Luo, ${ }^{1 \#}$ Yalin Shen, ${ }^{1}$ Changcheng Li, ${ }^{1}$ Yibo

Zhu, ${ }^{1}$ Qin Huang, ${ }^{1}$ Xingyu Mou,,${ }^{1}$ Xinyue Tang, ${ }^{1}$ Tonggen Liu, ${ }^{1}$ Siying Wu, ${ }^{3}$ Aiping Tong, ${ }^{1}$ Yongxing $\mathrm{He},{ }^{2 *}$ Rui Bao ${ }^{1 *}$

${ }^{1}$ Center of Infectious Diseases, State Key Laboratory of Biotherapy, West China

Hospital, Sichuan University and Collaborative Innovation Center, Chengdu 610093,

China;

${ }^{2}$ Ministry of Education Key Laboratory of Cell Activities and Stress Adaptations, School of Life Sciences, Lanzhou University, Lanzhou 730000, P.R. China;

${ }^{3}$ Department of Laboratory medicine, West China Hospital, Sichuan University, Chengdu 610044, China

\#These authors contributed equally: Yingjie Song, Siping Zhang and Guihua Luo. *Correspondence should be addressed to e-mail: Rui Bao: baorui@scu.edu.cn (R.B.); Yongxing He: heyx@1zu.edu.cn (Y.-X.H.) 


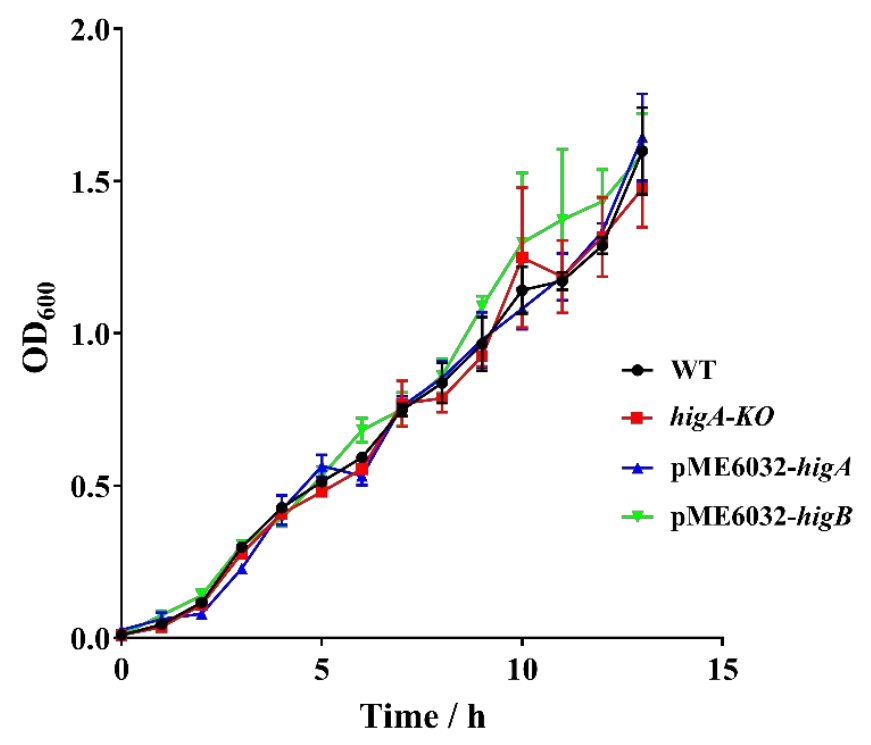

Figure S1. Growth curved of Pseudomonas aeruginosa PA14 and higA-KO in LB.

The strains containing complementation plasmid pME6032-higA and pME6032-higB were also measured and shown no significant difference with the WT. Data are represent of three independent experiments $( \pm \mathrm{SEM})$. 


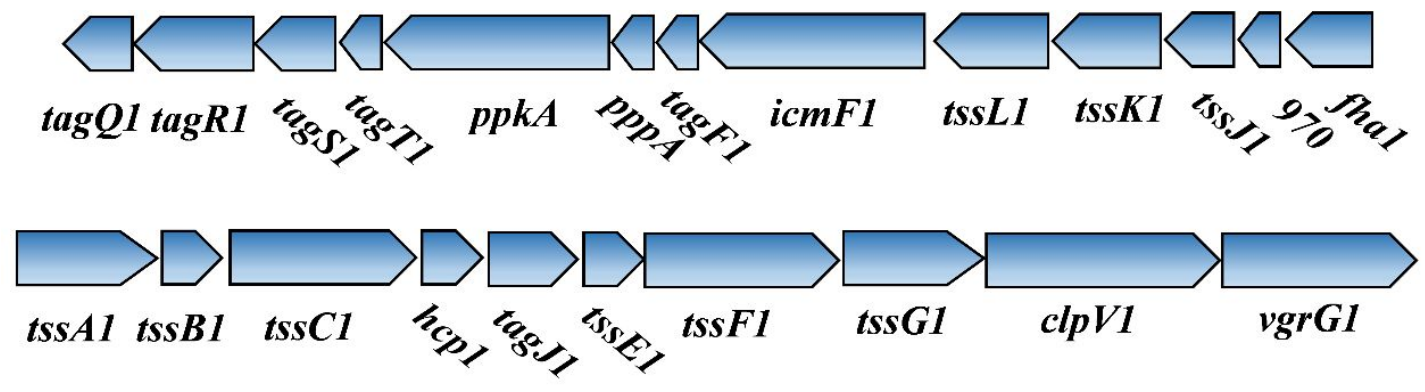

Figure S2. Diagram of the H1-T6SS operon. Representation of the H1-T6SS genomic Organization in Pseudomonas aeruginosa PAO1. 



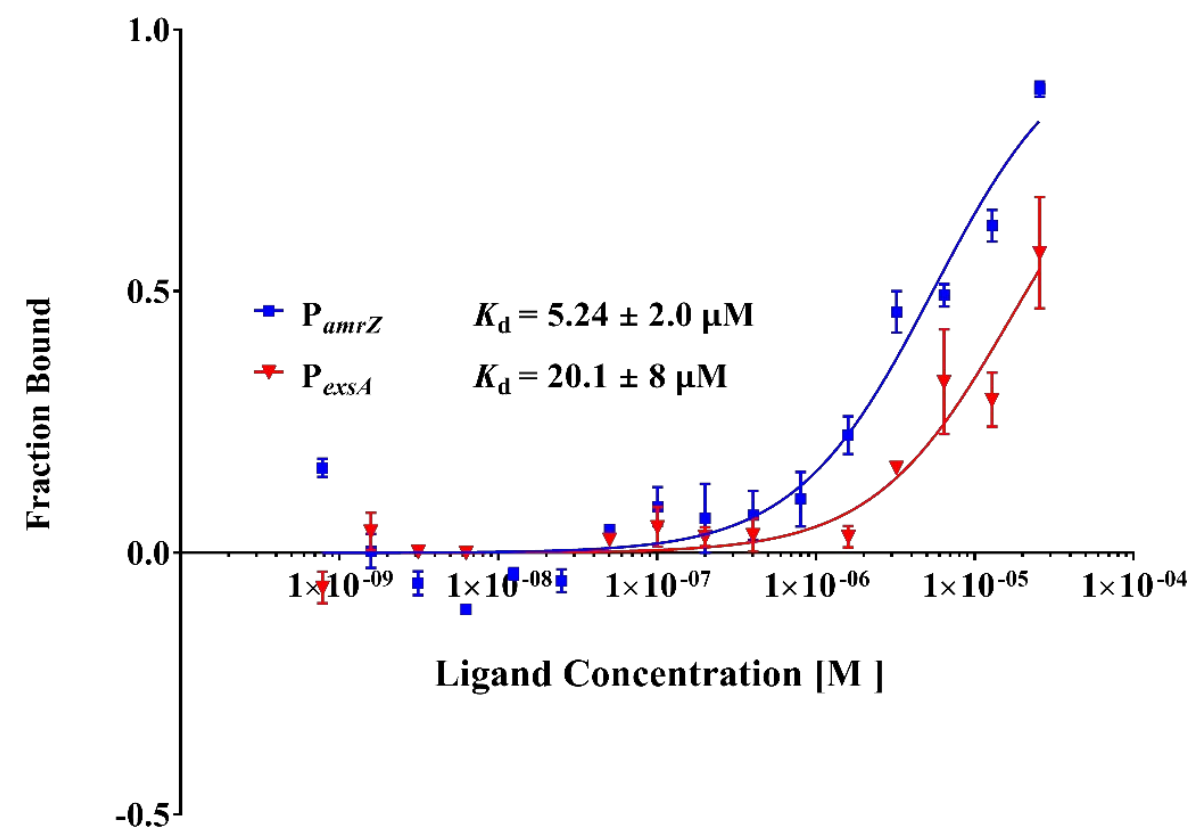

Figure S3. The interactions of HigA with $\operatorname{amr} Z$ and $\operatorname{exs} A$ operator were measured with MST. The final protein concentration was $100 \mathrm{nM}$ and double-stranded DNA have 16 doubling dilutions started from $50 \mu \mathrm{M}$. Each data point was calculated from three independent thermophoresis measurements. 


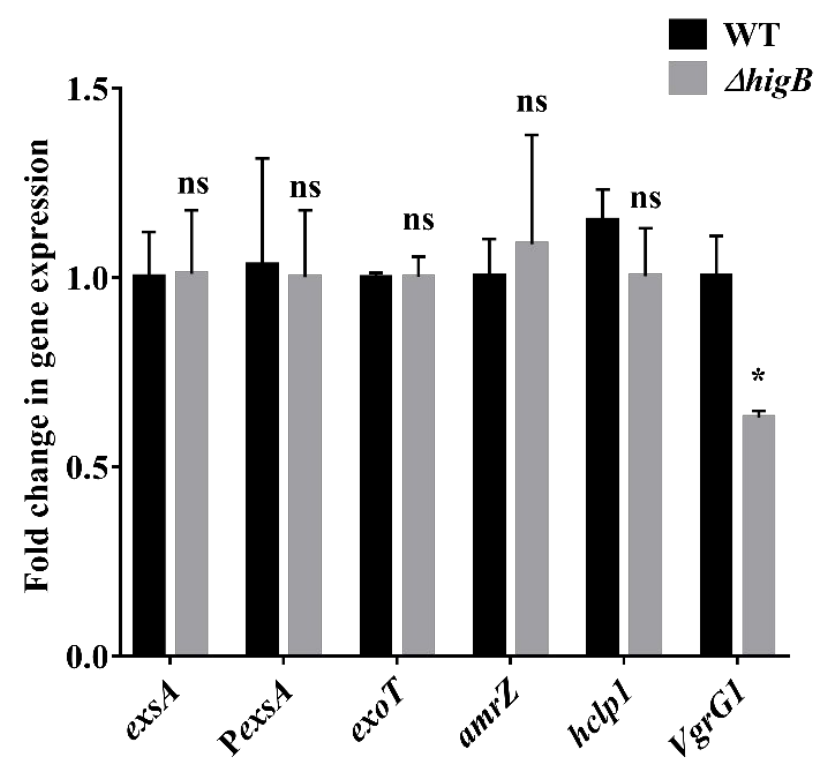

Figure S4. Relative mRNA levels of $\operatorname{exs} A$, exoT, amrZ, hcp1 and vgrG1 in 4 higB compared with Pseudomonas aeruginosa WT. The experiments were repeated three times and the error bars were shown. Significance was determined by student's t-test, ${ }^{*} p<0.05 ; * * p<0.01$ 



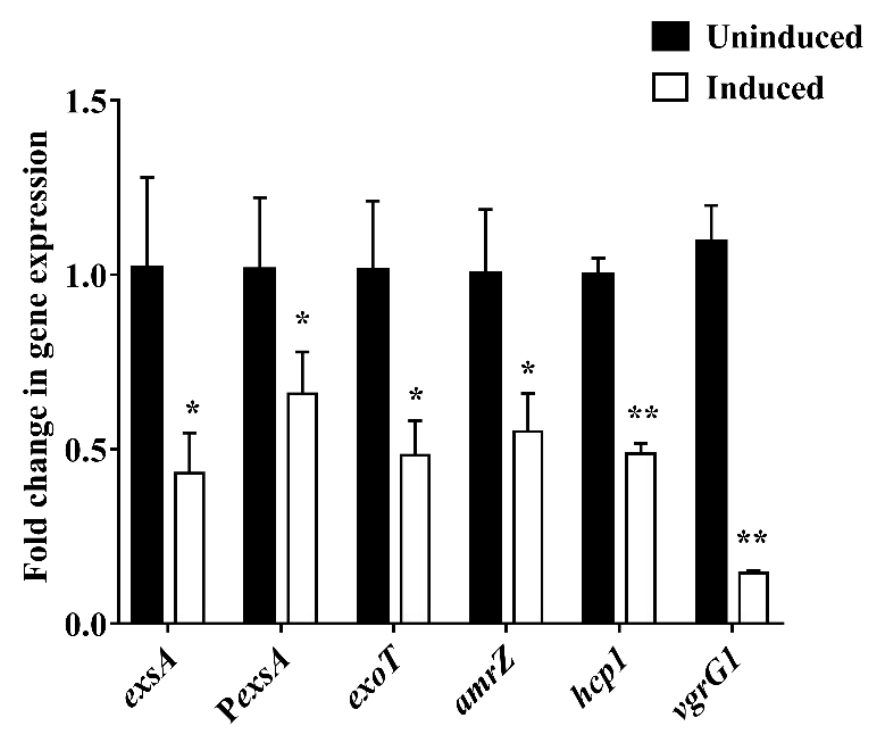

Figure S5. The effects of excess HigA on expression levels of exs A, exoT, amrZ, hcpl and $\operatorname{vgrG1}$. This was conducted by introducing a complementary plasmid pME6032-higA in the $\triangle$ higB $\triangle$ higA strain, and the expression of HigA was induced by adding $0.1 \mathrm{mM}$ IPTG for 3 hours. The experiments were repeated three times and the error bars were shown. Significance was determined by student's t-test, ${ }^{*} p<0.05 ;{ }^{* *} p$ $<0.01$. 

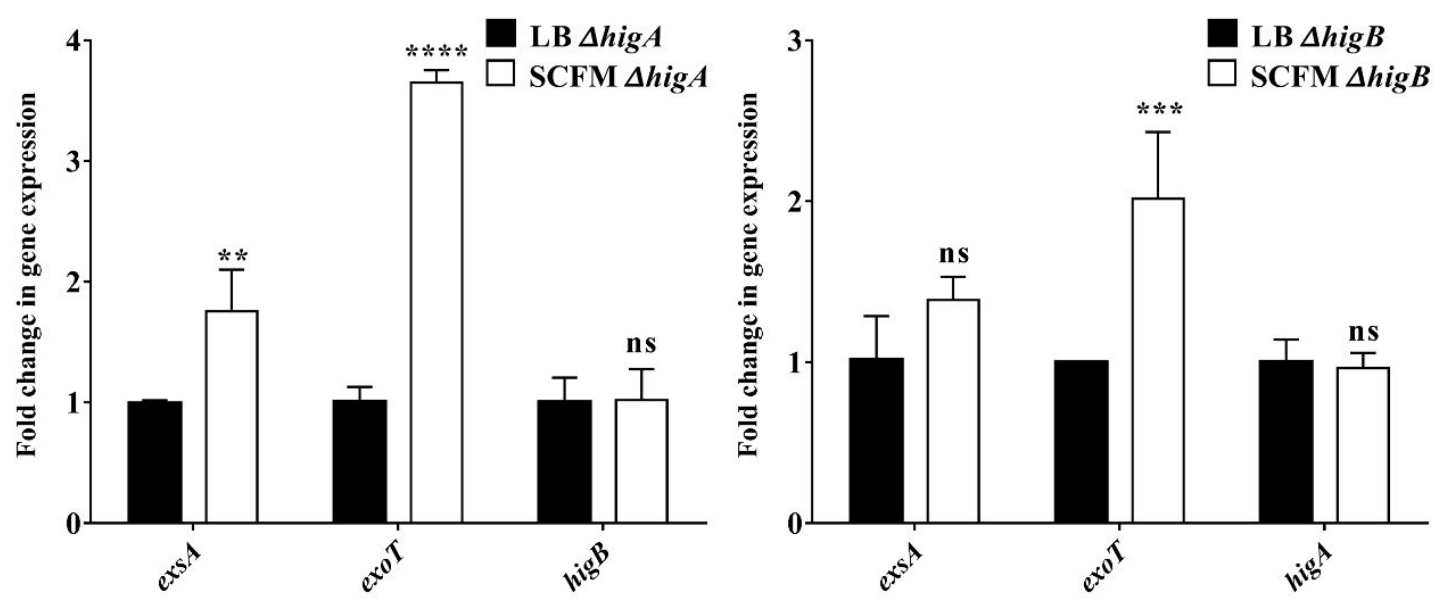

Figure S6. The effects of higB and $h i g A$ on expression levels of T3SS in SCFM

medium. The $\triangle h i g A$ and $\triangle h i g B$ strains were grown in SCFM and LB medium and the RNA was extracted for qRT-PCR. The experiments were repeated three times and the error bars were shown. Significance was determined by student's t-test, ${ }^{*} p<0.05 ;{ }^{*} p$ $<0.01$. 


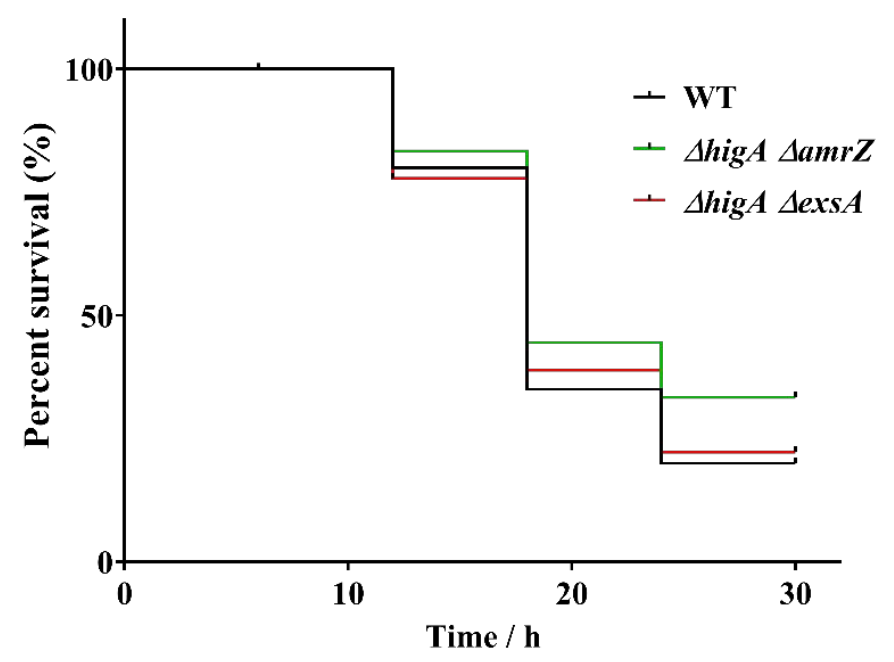

Figure S7. Survival analysis of $G$. mellonella larvae following injection of $P$. aeruginosa WT, $\Delta$ higA $A a m r Z$ and $\Delta$ hig $A \Delta \operatorname{exs} A$ strains. The larvae were monitored for 30 hours after the infection. (Mantel-Cox test for statistics, ${ }^{*}, \mathrm{P}<0.05$ ). 



\begin{tabular}{|c|c|c|c|c|c|c|c|c|c|c|}
\hline Replicon Name & Start & End & Strand & Search Pattern & Sequence & Feature Name & Feature Type & Feature Start & Feature End & Feature Strand \\
\hline Pseudomonas aeruginosa PAO1 & 225242 & 225251 & + & NTAACGNTAA & ATAACGATAA & & intergenic & & & \\
\hline Pseudomonas aeruginosa PAO1 & 1087474 & 1087483 & + & NTAACGNTAA & TTAACGTTAA & & intergenic & & & \\
\hline Pseudomonas aeruginosa PAO1 & 1087474 & 1087483 & - & NTAACGNTAA & TTAACGTTAA & & intergenic & & & \\
\hline Pseudomonas aeruginosa PAO1 & 1117641 & 1117650 & + & NTAACGNTAA & ATAACGATAA & & intergenic & & & \\
\hline Pseudomonas aeruginosa PAO1 & 1665090 & 1665099 & - & NTAACGNTAA & ATAACGATAA & zipA & gene & 1665065 & 1665934 & + \\
\hline Pseudomonas aeruginosa PAO1 & 1666090 & 1666099 & - & NTAACGNTAA & GTAACGGTAA & $\operatorname{lig}$ & gene & 1666025 & 1668409 & + \\
\hline Pseudomonas aeruginosa PAO1 & 1723297 & 1723306 & - & NTAACGNTAA & GTAACGATAA & $\operatorname{sdhB}$ & gene & 1723280 & 1723987 & + \\
\hline Pseudomonas aeruginosa PAO1 & 2544919 & 2544928 & + & NTAACGNTAA & GTAACGGTAA & $\mathrm{ambB}$ & gene & 2541197 & 2544946 & - \\
\hline Pseudomonas aeruginosa PAO1 & 2556300 & 2556309 & - & NTAACGNTAA & ATAACGGTAA & PA2318 & gene & 2556293 & 2556658 & + \\
\hline Pseudomonas aeruginosa PAO1 & 3483666 & 3483675 & + & NTAACGNTAA & ATAACGATAA & $\mathrm{xcpP}$ & gene & 3483642 & 3484349 & + \\
\hline Pseudomonas aeruginosa PAO1 & 3530112 & 3530121 & - & NTAACGNTAA & TTAACGGTAA & wbpK & gene & 3529508 & 3530458 & - \\
\hline Pseudomonas aeruginosa PAOI & 3683919 & 3683928 & - & NTAACGNTAA & ATAACGATAA & tle 1 & gene & 3681500 & 3684148 & - \\
\hline Pseudomonas aeruginosa PAOI & 3893261 & 3893270 & + & NTAACGNTAA & CTAACGGTAA & & intergenic & & & \\
\hline Pseudomonas aeruginosa PAO1 & 4033928 & 4033937 & + & NTAACGNTAA & GTAACGGTAA & PA3598 & gene & 4033850 & 4034665 & - \\
\hline Pseudomonas aeruginosa PAO1 & 4075157 & 4075166 & + & NTAACGNTAA & TTAACGATAA & dnaE & gene & 4075157 & 4078678 & - \\
\hline Pseudomonas aeruginosa PAO1 & 4609488 & 4609497 & + & NTAACGNTAA & ATAACGTTAA & & intergenic & & & \\
\hline Pseudomonas aeruginosa PAO1 & 4663708 & 4663717 & + & NTAACGNTAA & ATAACGATAA & & intergenic & & & \\
\hline Pseudomonas aeruginosa PAO1 & 5242860 & 5242869 & + & NTAACGNTAA & TTAACGTTAA & & intergenic & & & \\
\hline Pseudomonas aeruginosa PAO1 & 5242860 & 5242869 & - & NTAACGNTAA & TTAACGTTAA & & intergenic & & & \\
\hline Pseudomonas aeruginosa PAO1 & 5786723 & 5786732 & - & NTAACGNTAA & ATAACGATAA & & intergenic & & & \\
\hline Pseudomonas aeruginosa PAO1 & 722568 & 722577 & - & NTAACGTNAA & GTAACGTCAA & PA0668.1 & gene & 722096 & 723631 & + \\
\hline Pseudomonas aeruginosa PAO1 & 1060321 & 1060330 & - & NTAACGTNAA & CTAACGTAAA & & intergenic & & & \\
\hline Pseudomonas aeruginosa PAO1 & 1087474 & 1087483 & + & NTAACGTNAA & TTAACGTTAA & & intergenic & & & \\
\hline Pseudomonas aeruginosa PAO1 & 1087474 & 1087483 & - & NTAACGTNAA & TTAACGTTAA & & intergenic & & & \\
\hline Pseudomonas aeruginosa PAO1 & 2206830 & 2206839 & - & NTAACGTNAA & TTAACGTAAA & & intergenic & & & \\
\hline Pseudomonas aeruginosa PAO1 & 2206836 & 2206845 & + & NTAACGTNAA & TTAACGTAAA & & intergenic & & & \\
\hline Pseudomonas aeruginosa PAO1 & 2892161 & 2892170 & - & NTAACGTNAA & TTAACGTAAA & & intergenic & & & \\
\hline Pseudomonas aeruginosa PAO1 & 2892167 & 2892176 & + & NTAACGTNAA & TTAACGTAAA & & intergenic & & & \\
\hline Pseudomonas aeruginosa PAO1 & 3434013 & 3434022 & + & NTAACGTNAA & TTAACGTCAA & & intergenic & & & \\
\hline Pseudomonas aeruginosa PAO1 & 3751828 & 3751837 & - & NTAACGTNAA & CTAACGTGAA & & intergenic & & & \\
\hline Pseudomonas aeruginosa PAO1 & 4609488 & 4609497 & + & NTAACGTNAA & ATAACGTTAA & & intergenic & & & \\
\hline Pseudomonas aeruginosa PAO1 & 4793251 & 4793260 & + & NTAACGTNAA & GTAACGTCAA & PA4280.5 & gene & 4792196 & 4793731 & - \\
\hline Pseudomonas aeruginosa PAO1 & 4974135 & 4974144 & + & NTAACGTNAA & TTAACGTAAA & & intergenic & & & \\
\hline Pseudomonas aeruginosa PAO1 & 5067823 & 5067832 & + & NTAACGTNAA & GTAACGTCAA & & intergenic & & & \\
\hline Pseudomonas aeruginosa PAO1 & 5242860 & 5242869 & + & NTAACGTNAA & TTAACGTTAA & & intergenic & & & \\
\hline Pseudomonas aeruginosa PAO1 & 5242860 & 5242869 & - & NTAACGTNAA & TTAACGTTAA & & intergenic & & & \\
\hline Pseudomonas aeruginosa PAO1 & 5268779 & 5268788 & + & NTAACGTNAA & GTAACGTCAA & PA4690.5 & gene & 5267724 & 5269259 & - \\
\hline Pseudomonas aeruginosa PAO1 & 5349038 & 5349047 & - & NTAACGTNAA & GTAACGTCAA & dnaK & gene & 5347198 & 5349111 & - \\
\hline
\end{tabular}

Table S1. Potential HigA-binding loci in Pseudomonas aeruginosa PAO1 genome. 


\begin{tabular}{|c|c|c|c|c|c|c|c|c|c|c|}
\hline Pseudomonas aeruginosa PAO1 & 5926870 & 5926879 & + & NTAACGTNAA & ATAACGTGAA & PA5264 & gene & 5926134 & 5927105 & - \\
\hline Pseudomonas aeruginosa PAO1 & 6044263 & 6044272 & + & NTAACGTNAA & GTAACGTCAA & PA5369.5 & gene & 6043208 & 6044743 & - \\
\hline Pseudomonas aeruginosa PAO1 & 255360 & 255369 & + & NTAACGTTAN & CTAACGTTAT & & intergenic & & & \\
\hline Pseudomonas aeruginosa PAO1 & 255360 & 255369 & - & NTAACGTTAN & ATAACGTTAG & & intergenic & & & \\
\hline Pseudomonas aeruginosa PAO1 & 1087474 & 1087483 & + & NTAACGTTAN & TTAACGTTAA & & intergenic & & & \\
\hline Pseudomonas aeruginosa PAO1 & 1087474 & 1087483 & - & NTAACGTTAN & TTAACGTTAA & & intergenic & & & \\
\hline Pseudomonas aeruginosa $\mathrm{PAO} 1$ & 2472387 & 2472396 & + & NTAACGTTAN & GTAACGTTAC & pslO & gene & 2472104 & 2472409 & + \\
\hline Pseudomonas aeruginosa PAO1 & 2472387 & 2472396 & - & NTAACGTTAN & GTAACGTTAC & pslO & gene & 2472104 & 2472409 & + \\
\hline Pseudomonas aeruginosa PAO1 & 2472411 & 2472420 & + & NTAACGTTAN & GTAACGTTAC & & intergenic & & & \\
\hline Pseudomonas aeruginosa $\mathrm{PAO1}$ & 2472411 & 2472420 & - & NTAACGTTAN & GTAACGTTAC & & intergenic & & & \\
\hline Pseudomonas aeruginosa PAO1 & 4609488 & 4609497 & + & NTAACGTTAN & ATAACGTTAA & & intergenic & & & \\
\hline Pseudomonas aeruginosa PAO1 & 4609488 & 4609497 & - & NTAACGTTAN & TTAACGTTAT & & intergenic & & & \\
\hline Pseudomonas aeruginosa PAO1 & 5242860 & 5242869 & + & NTAACGTTAN & TTAACGTTAA & & intergenic & & & \\
\hline Pseudomonas aeruginosa PAO1 & 5242860 & 5242869 & - & NTAACGTTAN & TTAACGTTAA & & intergenic & & & \\
\hline Pseudomonas aeruginosa PAO1 & 54649 & 54658 & + & NTAACGTTNA & GTAACGTTCA & & intergenic & & & \\
\hline Pseudomonas aeruginosa PAO1 & 54860 & 54869 & + & NTAACGTTNA & GTAACGTTCA & & intergenic & & & \\
\hline Pseudomonas aeruginosa PAO1 & 298459 & 298468 & + & NTAACGTTNA & CTAACGTTGA & & intergenic & & & \\
\hline Pseudomonas aeruginosa PAO1 & 1087474 & 1087483 & + & NTAACGTTNA & TTAACGTTAA & & intergenic & & & \\
\hline Pseudomonas aeruginosa PAO1 & 1087474 & 1087483 & - & NTAACGTTNA & TTAACGTTAA & & intergenic & & & \\
\hline Pseudomonas aeruginosa PAO1 & 2291767 & 2291776 & - & NTAACGTTNA & GTAACGTTGA & & intergenic & & & \\
\hline Pseudomonas aeruginosa PAO1 & 2756670 & 2756679 & - & NTAACGTTNA & GTAACGTTTA & & intergenic & & & \\
\hline Pseudomonas aeruginosa PAO1 & 3498684 & 3498693 & + & NTAACGTTNA & GTAACGTTCA & PA3116 & gene & 3498374 & 3499384 & - \\
\hline Pseudomonas aeruginosa PAO1 & 4609488 & 4609497 & + & NTAACGTTNA & ATAACGTTAA & & intergenic & & & \\
\hline Pseudomonas aeruginosa PAO1 & 5172427 & 5172436 & + & NTAACGTTNA & ATAACGTTGA & mscL & gene & 5172253 & 5172666 & + \\
\hline Pseudomonas aeruginosa PAO1 & 5220497 & 5220506 & - & NTAACGTTNA & GTAACGTTGA & cupE6 & gene & 5219938 & 5220885 & + \\
\hline Pseudomonas aeruginosa PAO1 & 5242860 & 5242869 & + & NTAACGTTNA & TTAACGTTAA & & intergenic & & & \\
\hline Pseudomonas aeruginosa PAO1 & 5242860 & 5242869 & - & NTAACGTTNA & TTAACGTTAA & & intergenic & & & \\
\hline Pseudomonas aeruginosa PAO1 & 5900670 & 5900679 & + & NTAACGTTNA & GTAACGTTGA & $\operatorname{trxA}$ & gene & 5900369 & 5900695 & - \\
\hline Pseudomonas aeruginosa PAO1 & 123740 & 123749 & - & TNAACGNTAA & TCAACGCTAA & & intergenic & & & \\
\hline Pseudomonas aeruginosa PAO1 & 1069111 & 1069120 & + & TNAACGNTAA & TCAACGGTAA & PA0987 & gene & 1068489 & 1069331 & + \\
\hline Pseudomonas aeruginosa PAO1 & 1087474 & 1087483 & + & TNAACGNTAA & TTAACGTTAA & & intergenic & & & \\
\hline Pseudomonas aeruginosa PAO1 & 1087474 & 1087483 & - & TNAACGNTAA & TTAACGTTAA & & intergenic & & & \\
\hline Pseudomonas aeruginosa PAO1 & 1467867 & 1467876 & - & TNAACGNTAA & TCAACGGTAA & PA1353 & gene & 1467488 & 1467901 & - \\
\hline Pseudomonas aeruginosa PAO1 & 3120854 & 3120863 & + & TNAACGNTAA & TCAACGGTAA & oprQ & gene & 3120073 & 3121350 & + \\
\hline Pseudomonas aeruginosa PAO1 & 3530112 & 3530121 & - & TNAACGNTAA & TTAACGGTAA & wbpK & gene & 3529508 & 3530458 & - \\
\hline Pseudomonas aeruginosa PAO1 & 4075157 & 4075166 & + & TNAACGNTAA & TTAACGATAA & dnaE & gene & 4075157 & 4078678 & - \\
\hline Pseudomonas aeruginosa PAO1 & 5120078 & 5120087 & + & TNAACGNTAA & TGAACGGTAA & PA4571 & gene & 5118538 & 5120565 & + \\
\hline Pseudomonas aeruginosa PAO1 & 5242860 & 5242869 & + & TNAACGNTAA & TTAACGTTAA & & intergenic & & & \\
\hline Pseudomonas aeruginosa PAO1 & 5242860 & 5242869 & - & TNAACGNTAA & TTAACGTTAA & & intergenic & & & \\
\hline Pseudomonas aeruginosa PAO1 & 5680727 & 5680736 & - & TNAACGNTAA & TGAACGCTAA & & intergenic & & & \\
\hline Pseudomonas aeruginosa PAO1 & 117466 & 117475 & + & TNAACGTNAA & TCAACGTCAA & PA0095 & gene & 115299 & 117524 & + \\
\hline Pseudomonas aeruginosa PAO1 & 120489 & 120498 & + & TNAACGTNAA & TGAACGTCAA & PA0099 & gene & 120164 & 121324 & + \\
\hline Pseudomonas aeruginosa PAO1 & 173322 & 173331 & + & TNAACGTNAA & TCAACGTGAA & PA0151 & gene & 171047 & 173434 & + \\
\hline
\end{tabular}




\begin{tabular}{|c|c|c|c|c|c|c|c|c|c|c|}
\hline Pseudomonas aeruginosa PAO1 & 196267 & 196276 & - & TNAACGTNAA & TCAACGTCAA & siaA & gene & 194757 & 196748 & - \\
\hline Pseudomonas aeruginosa $\mathrm{PAO1}$ & 230438 & 230447 & + & TNAACGTNAA & TCAACGTGAA & PA0201 & gene & 229954 & 230535 & + \\
\hline Pseudomonas aeruginosa PAO1 & 283036 & 283045 & - & TNAACGTNAA & TGAACGTCAA & PA0251 & gene & 282912 & 283553 & - \\
\hline Pseudomonas aeruginosa PAO1 & 350966 & 350975 & + & TNAACGTNAA & TCAACGTCAA & PA0310 & gene & 350890 & 351588 & + \\
\hline Pseudomonas aeruginosa PAO1 & 439392 & 439401 & - & TNAACGTNAA & TGAACGTCAA & PA0397 & gene & 438938 & 439837 & - \\
\hline Pseudomonas aeruginosa PAO1 & 483550 & 483559 & - & TNAACGTNAA & TCAACGTCAA & sahH & gene & 482706 & 484115 & - \\
\hline Pseudomonas aeruginosa $\mathrm{PAO} 1$ & 579130 & 579139 & - & TNAACGTNAA & TCAACGTGAA & nirS & gene & 578394 & 580100 & - \\
\hline Pseudomonas aeruginosa PAO1 & 613405 & 613414 & + & TNAACGTNAA & TCAACGTCAA & fda & gene & 613338 & 614402 & + \\
\hline Pseudomonas aeruginosa PAO1 & 653768 & 653777 & - & TNAACGTNAA & TCAACGTGAA & surA & gene & 652480 & 653772 & - \\
\hline Pseudomonas aeruginosa PAO1 & 669953 & 669962 & + & TNAACGTNAA & TCAACGTCAA & rpe & gene & 669415 & 670089 & + \\
\hline Pseudomonas aeruginosa PAO1 & 933799 & 933808 & - & TNAACGTNAA & TGAACGTCAA & fumC2 & gene & 932718 & 934112 & - \\
\hline Pseudomonas aeruginosa PAO1 & 953767 & 953776 & + & TNAACGTNAA & TCAACGTCAA & phhR & gene & 953691 & 955250 & + \\
\hline Pseudomonas aeruginosa PAO1 & 1056995 & 1057004 & + & TNAACGTNAA & TGAACGTGAA & tolB & gene & 1056049 & 1057347 & + \\
\hline Pseudomonas aeruginosa PAO1 & 1087474 & 1087483 & + & TNAACGTNAA & TTAACGTTAA & & intergenic & & & \\
\hline Pseudomonas aeruginosa PAO1 & 1087474 & 1087483 & - & TNAACGTNAA & TTAACGTTAA & & intergenic & & & \\
\hline Pseudomonas aeruginosa PAO1 & 1365985 & 1365994 & - & TNAACGTNAA & TCAACGTCAA & lhpM & gene & 1365873 & 1366538 & - \\
\hline Pseudomonas aeruginosa PAO1 & 1583477 & 1583486 & + & TNAACGTNAA & TGAACGTGAA & flhF & gene & 1582528 & 1583817 & + \\
\hline Pseudomonas aeruginosa PAO1 & 1588926 & 1588935 & + & TNAACGTNAA & TCAACGTCAA & PA1458 & gene & 1587023 & 1589284 & + \\
\hline Pseudomonas aeruginosa PAO1 & 1590050 & 1590059 & + & TNAACGTNAA & TCAACGTCAA & PA1459 & gene & 1589338 & 1590444 & + \\
\hline Pseudomonas aeruginosa PAO1 & 1751006 & 1751015 & - & TNAACGTNAA & TCAACGTCAA & PA1608 & gene & 1749895 & 1751520 & - \\
\hline Pseudomonas aeruginosa $\mathrm{PAO} 1$ & 1796481 & 1796490 & + & TNAACGTNAA & TCAACGTGAA & PA1649 & gene & 1796138 & 1796899 & + \\
\hline Pseudomonas aeruginosa PAO1 & 1880016 & 1880025 & + & TNAACGTNAA & TCAACGTCAA & PA1736 & gene & 1878980 & 1880185 & + \\
\hline Pseudomonas aeruginosa PAO1 & 1906725 & 1906734 & + & TNAACGTNAA & TCAACGTCAA & PA1763 & gene & 1905797 & 1906822 & + \\
\hline Pseudomonas aeruginosa PAO1 & 2120385 & 2120394 & - & TNAACGTNAA & TCAACGTGAA & PA1939 & gene & 2120225 & 2122222 & - \\
\hline Pseudomonas aeruginosa PAO1 & 2188077 & 2188086 & + & TNAACGTNAA & TCAACGTCAA & atoB & gene & 2187065 & 2188246 & + \\
\hline Pseudomonas aeruginosa PAO1 & 2206830 & 2206839 & - & TNAACGTNAA & TTAACGTAAA & & intergenic & & & \\
\hline Pseudomonas aeruginosa PAO1 & 2206836 & 2206845 & + & TNAACGTNAA & TTAACGTAAA & & intergenic & & & \\
\hline Pseudomonas aeruginosa PAO1 & 2412557 & 2412566 & + & TNAACGTNAA & TGAACGTCAA & hcnA & gene & 2412546 & 2412860 & + \\
\hline Pseudomonas aeruginosa $\mathrm{PAO} 1$ & 2563855 & 2563864 & + & TNAACGTNAA & TCAACGTCAA & gapN & gene & 2562447 & 2564072 & + \\
\hline Pseudomonas aeruginosa PAO1 & 2625663 & 2625672 & + & TNAACGTNAA & TCAACGTCAA & $\operatorname{vgrG} 3$ & gene & 2624204 & 2626210 & + \\
\hline Pseudomonas aeruginosa $\mathrm{PAO} 1$ & 2657522 & 2657531 & + & TNAACGTNAA & TCAACGTCAA & fpvA & gene & 2655187 & 2657634 & + \\
\hline Pseudomonas aeruginosa PAO1 & 2756845 & 2756854 & + & TNAACGTNAA & TCAACGTGAA & & intergenic & & & \\
\hline Pseudomonas aeruginosa PAO1 & 2810493 & 2810502 & + & TNAACGTNAA & TCAACGTGAA & $\operatorname{mexF}$ & gene & 2810009 & 2813197 & + \\
\hline Pseudomonas aeruginosa PAO1 & 2855014 & 2855023 & - & TNAACGTNAA & TGAACGTCAA & muxA & gene & 2854011 & 2855291 & - \\
\hline Pseudomonas aeruginosa PAO1 & 2874311 & 2874320 & - & TNAACGTNAA & TCAACGTCAA & PA2543 & gene & 2873824 & 2875563 & - \\
\hline Pseudomonas aeruginosa PAO1 & 2892161 & 2892170 & - & TNAACGTNAA & TTAACGTAAA & & intergenic & & & \\
\hline Pseudomonas aeruginosa PAO1 & 2892167 & 2892176 & + & TNAACGTNAA & TTAACGTAAA & & intergenic & & & \\
\hline Pseudomonas aeruginosa PAO1 & 2933649 & 2933658 & - & TNAACGTNAA & TGAACGTGAA & vqsR & gene & 2933582 & 2934388 & - \\
\hline Pseudomonas aeruginosa PAO1 & 2991965 & 2991974 & + & TNAACGTNAA & TCAACGTGAA & nuol & gene & 2991442 & 2991990 & + \\
\hline Pseudomonas aeruginosa PAO1 & 3320689 & 3320698 & - & TNAACGTNAA & TGAACGTGAA & holB & gene & 3320064 & 3321050 & - \\
\hline Pseudomonas aeruginosa PAO1 & 3431832 & 3431841 & - & TNAACGTNAA & TGAACGTCAA & pelA & gene & 3431045 & 3433891 & - \\
\hline Pseudomonas aeruginosa PAO1 & 3434013 & 3434022 & + & TNAACGTNAA & TTAACGTCAA & & intergenic & & & \\
\hline Pseudomonas aeruginosa PAO1 & 3477970 & 3477979 & - & TNAACGTNAA & TGAACGTGAA & $\mathrm{xcpX}$ & gene & 3477627 & 3478628 & - \\
\hline
\end{tabular}




\begin{tabular}{|c|c|c|c|c|c|c|c|c|c|c|}
\hline Pseudomonas aeruginosa PAO1 & 3563916 & 3563925 & + & TNAACGTNAA & TGAACGTCAA & PA3173 & gene & 3563546 & 3564286 & + \\
\hline Pseudomonas aeruginosa PAO1 & 3570139 & 3570148 & - & TNAACGTNAA & TCAACGTCAA & PA3179 & gene & 3569106 & 3570266 & - \\
\hline Pseudomonas aeruginosa PAO1 & 3762526 & 3762535 & + & TNAACGTNAA & TCAACGTCAA & PA3350 & gene & 3761961 & 3762659 & + \\
\hline Pseudomonas aeruginosa PAO1 & 3804696 & 3804705 & - & TNAACGTNAA & TCAACGTGAA & PA3399 & gene & 3804545 & 3804886 & - \\
\hline Pseudomonas aeruginosa PAO1 & 3944861 & 3944870 & - & TNAACGTNAA & TCAACGTGAA & $\operatorname{argG}$ & gene & 3944662 & 3945879 & - \\
\hline Pseudomonas aeruginosa $\mathrm{PAO1}$ & 3965855 & 3965864 & + & TNAACGTNAA & TCAACGTCAA & alg44 & gene & 3965842 & 3967011 & + \\
\hline Pseudomonas aeruginosa $\mathrm{PAO} 1$ & 3973243 & 3973252 & + & TNAACGTNAA & TCAACGTCAA & algL & gene & 3973014 & 3974117 & + \\
\hline Pseudomonas aeruginosa PAO1 & 4060896 & 4060905 & - & TNAACGTNAA & TCAACGTCAA & surE & gene & 4060625 & 4061374 & - \\
\hline Pseudomonas aeruginosa PAO1 & 4062511 & 4062520 & - & TNAACGTNAA & TCAACGTCAA & ygbB & gene & 4062426 & 4062899 & - \\
\hline Pseudomonas aeruginosa $\mathrm{PAO1}$ & 4215252 & 4215261 & - & TNAACGTNAA & TGAACGTAAA & & intergenic & & & \\
\hline Pseudomonas aeruginosa PAO1 & 4298546 & 4298555 & + & TNAACGTNAA & TCAACGTCAA & PA3837 & gene & 4298311 & 4299201 & + \\
\hline Pseudomonas aeruginosa PAO1 & 4560306 & 4560315 & + & TNAACGTNAA & TAAACGTGAA & сupB6 & gene & 4560295 & 4561440 & - \\
\hline Pseudomonas aeruginosa PAO1 & 4560674 & 4560683 & - & TNAACGTNAA & TCAACGTCAA & cupB6 & gene & 4560295 & 4561440 & - \\
\hline Pseudomonas aeruginosa PAO1 & 4642063 & 4642072 & + & TNAACGTNAA & TGAACGTCAA & PA4148 & gene & 4641711 & 4642511 & + \\
\hline Pseudomonas aeruginosa PAO1 & 4668515 & 4668524 & + & TNAACGTNAA & TCAACGTCAA & PA4172 & gene & 4668490 & 4669290 & + \\
\hline Pseudomonas aeruginosa PAO1 & 4702892 & 4702901 & - & TNAACGTNAA & TCAACGTCAA & nmoA & gene & 4702042 & 4703097 & - \\
\hline Pseudomonas aeruginosa PAO1 & 4724745 & 4724754 & - & TNAACGTNAA & TCAACGTCAA & fptA & gene & 4724639 & 4726801 & - \\
\hline Pseudomonas aeruginosa PAO1 & 4750953 & 4750962 & + & TNAACGTNAA & TCAACGTCAA & uvrA & gene & 4748756 & 4751593 & + \\
\hline Pseudomonas aeruginosa PAO1 & 4756214 & 4756223 & - & TNAACGTNAA & TCAACGTCAA & rpsK & gene & 4756084 & 4756473 & - \\
\hline Pseudomonas aeruginosa PAO1 & 4765825 & 4765834 & - & TNAACGTNAA & TGAACGTCAA & $\mathrm{rplW}$ & gene & 4765713 & 4766012 & - \\
\hline Pseudomonas aeruginosa PAO1 & 4787808 & 4787817 & - & TNAACGTNAA & TCAACGTCAA & birA & gene & 4787477 & 4788415 & - \\
\hline Pseudomonas aeruginosa PAO1 & 4815299 & 4815308 & + & TNAACGTNAA & TCAACGTGAA & PA4292 & gene & 4815043 & 4816512 & + \\
\hline Pseudomonas aeruginosa $\mathrm{PAO} 1$ & 4821593 & 4821602 & - & TNAACGTNAA & TCAACGTCAA & $\operatorname{tadG}$ & gene & 4821385 & 4823055 & - \\
\hline Pseudomonas aeruginosa PAO1 & 4821680 & 4821689 & - & TNAACGTNAA & TCAACGTGAA & $\operatorname{tadG}$ & gene & 4821385 & 4823055 & - \\
\hline Pseudomonas aeruginosa PAO1 & 4974135 & 4974144 & + & TNAACGTNAA & TTAACGTAAA & & intergenic & & & \\
\hline Pseudomonas aeruginosa PAO1 & 5070862 & 5070871 & + & TNAACGTNAA & TCAACGTCAA & pilB & gene & 5069763 & 5071463 & + \\
\hline Pseudomonas aeruginosa PAO1 & 5085486 & 5085495 & + & TNAACGTNAA & TCAACGTAAA & lepA & gene & 5082443 & 5086696 & + \\
\hline Pseudomonas aeruginosa PAO1 & 5242860 & 5242869 & + & TNAACGTNAA & TTAACGTTAA & & intergenic & & & \\
\hline Pseudomonas aeruginosa PAO1 & 5242860 & 5242869 & - & TNAACGTNAA & TTAACGTTAA & & intergenic & & & \\
\hline Pseudomonas aeruginosa PAO1 & 5348216 & 5348225 & - & TNAACGTNAA & TGAACGTCAA & dnaK & gene & 5347198 & 5349111 & - \\
\hline Pseudomonas aeruginosa PAO1 & 5372748 & 5372757 & - & TNAACGTNAA & TCAACGTCAA & PA4785 & gene & 5372591 & 5373868 & - \\
\hline Pseudomonas aeruginosa PAO1 & 5375060 & 5375069 & + & TNAACGTNAA & TCAACGTCAA & PA4786 & gene & 5374123 & 5375478 & + \\
\hline Pseudomonas aeruginosa PAO1 & 5427819 & 5427828 & - & TNAACGTNAA & TGAACGTCAA & cntO & gene & 5427716 & 5429842 & - \\
\hline Pseudomonas aeruginosa PAO1 & 5623827 & 5623836 & - & TNAACGTNAA & TGAACGTCAA & PA5005 & gene & 5623040 & 5624797 & - \\
\hline Pseudomonas aeruginosa PAO1 & 5799087 & 5799096 & - & TNAACGTNAA & TCAACGTCAA & PA5150 & gene & 5798693 & 5799430 & - \\
\hline Pseudomonas aeruginosa PAO1 & 5974266 & 5974275 & - & TNAACGTNAA & TCAACGTGAA & PA5306 & gene & 5974190 & 5974381 & - \\
\hline Pseudomonas aeruginosa PAO1 & 5974520 & 5974529 & - & TNAACGTNAA & TCAACGTCAA & PA5307 & gene & 5974402 & 5976969 & - \\
\hline Pseudomonas aeruginosa PAO1 & 6002137 & 6002146 & + & TNAACGTNAA & TGAACGTGAA & cre & gene & 6002121 & 6002900 & + \\
\hline Pseudomonas aeruginosa PAO1 & 54649 & 54658 & - & TNAACGTTAN & TGAACGTTAC & & intergenic & & & \\
\hline Pseudomonas aeruginosa PAO1 & 54860 & 54869 & - & TNAACGTTAN & TGAACGTTAC & & intergenic & & & \\
\hline Pseudomonas aeruginosa PAO1 & 298459 & 298468 & - & TNAACGTTAN & TCAACGTTAG & & intergenic & & & \\
\hline Pseudomonas aeruginosa PAO1 & 1087474 & 1087483 & + & TNAACGTTAN & TTAACGTTAA & & intergenic & & & \\
\hline Pseudomonas aeruginosa PAO1 & 1087474 & 1087483 & - & TNAACGTTAN & TTAACGTTAA & & intergenic & & & \\
\hline
\end{tabular}




\begin{tabular}{|c|c|c|c|c|c|c|c|c|c|c|}
\hline Pseudomonas aeruginosa PAO1 & 2291767 & 2291776 & + & TNAACGTTAN & TCAACGTTAC & & intergenic & & & \\
\hline Pseudomonas aeruginosa $\mathrm{PAO1}$ & 2756670 & 2756679 & + & TNAACGTTAN & TAAACGTTAC & & intergenic & & & \\
\hline Pseudomonas aeruginosa PAO1 & 3498684 & 3498693 & - & TNAACGTTAN & TGAACGTTAC & PA3116 & gene & 3498374 & 3499384 & - \\
\hline Pseudomonas aeruginosa $\mathrm{PAO1}$ & 4609488 & 4609497 & - & TNAACGTTAN & TTAACGTTAT & & intergenic & & & \\
\hline Pseudomonas aeruginosa $\mathrm{PAO} 1$ & 5172427 & 5172436 & - & TNAACGTTAN & TCAACGTTAT & $\mathrm{mscL}$ & gene & 5172253 & 5172666 & + \\
\hline Pseudomonas aeruginosa $\mathrm{PAO1}$ & 5220497 & 5220506 & + & TNAACGTTAN & TCAACGTTAC & cupE6 & gene & 5219938 & 5220885 & + \\
\hline Pseudomonas aeruginosa PAO1 & 5242860 & 5242869 & + & TNAACGTTAN & TTAACGTTAA & & intergenic & & & \\
\hline Pseudomonas aeruginosa PAO1 & 5242860 & 5242869 & - & TNAACGTTAN & TTAACGTTAA & & intergenic & & & \\
\hline Pseudomonas aeruginosa $\mathrm{PAO} 1$ & 5900670 & 5900679 & - & TNAACGTTAN & TCAACGTTAC & $\operatorname{trxA}$ & gene & 5900369 & 5900695 & - \\
\hline Pseudomonas aeruginosa PAO1 & 1087474 & 1087483 & + & TNAACGTTNA & TTAACGTTAA & & intergenic & & & \\
\hline Pseudomonas aeruginosa PAO1 & 1087474 & 1087483 & - & TNAACGTTNA & TTAACGTTAA & & intergenic & & & \\
\hline Pseudomonas aeruginosa PAO1 & 5242860 & 5242869 & + & TNAACGTTNA & TTAACGTTAA & & intergenic & & & \\
\hline Pseudomonas aeruginosa PAO1 & 5242860 & 5242869 & - & TNAACGTTNA & TTAACGTTAA & & intergenic & & & \\
\hline Pseudomonas aeruginosa PAO1 & 6051387 & 6051396 & + & TNAACGTTNA & TGAACGTTCA & & intergenic & & & \\
\hline Pseudomonas aeruginosa PAO1 & 6051387 & 6051396 & - & TNAACGTTNA & TGAACGTTCA & & intergenic & & & \\
\hline Pseudomonas aeruginosa PAO1 & 654516 & 654525 & + & TTANCGNTAA & TTACCGGTAA & lptD & gene & 653753 & 656527 & - \\
\hline Pseudomonas aeruginosa PAO1 & 654516 & 654525 & - & TTANCGNTAA & TTACCGGTAA & lptD & gene & 653753 & 656527 & - \\
\hline Pseudomonas aeruginosa PAO1 & 1087474 & 1087483 & + & TTANCGNTAA & TTAACGTTAA & & intergenic & & & \\
\hline Pseudomonas aeruginosa PAO1 & 1087474 & 1087483 & - & TTANCGNTAA & TTAACGTTAA & & intergenic & & & \\
\hline Pseudomonas aeruginosa PAO1 & 1299483 & 1299492 & + & TTANCGNTAA & TTATCGATAA & PA1197 & gene & 1298722 & 1299492 & + \\
\hline Pseudomonas aeruginosa $\mathrm{PAO} 1$ & 1299483 & 1299492 & - & TTANCGNTAA & TTATCGATAA & PA1197 & gene & 1298722 & 1299492 & + \\
\hline Pseudomonas aeruginosa PAO1 & 2120589 & 2120598 & + & TTANCGNTAA & TTATCGATAA & PA1939 & gene & 2120225 & 2122222 & - \\
\hline Pseudomonas aeruginosa PAO1 & 2120589 & 2120598 & - & TTANCGNTAA & TTATCGATAA & PA1939 & gene & 2120225 & 2122222 & - \\
\hline Pseudomonas aeruginosa PAO1 & 3119794 & 3119803 & + & TTANCGNTAA & TTATCGATAA & & intergenic & & & \\
\hline Pseudomonas aeruginosa PAO1 & 3119794 & 3119803 & - & TTANCGNTAA & TTATCGATAA & & intergenic & & & \\
\hline Pseudomonas aeruginosa PAO1 & 3530112 & 3530121 & + & TTANCGNTAA & TTACCGTTAA & wbpK & gene & 3529508 & 3530458 & - \\
\hline Pseudomonas aeruginosa PAO1 & 3530112 & 3530121 & - & TTANCGNTAA & TTAACGGTAA & wbpK & gene & 3529508 & 3530458 & - \\
\hline Pseudomonas aeruginosa PAO1 & 3937875 & 3937884 & + & TTANCGNTAA & TTATCGATAA & & intergenic & & & \\
\hline Pseudomonas aeruginosa $\mathrm{PAO} 1$ & 3937875 & 3937884 & - & TTANCGNTAA & TTATCGATAA & & intergenic & & & \\
\hline Pseudomonas aeruginosa PAO1 & 4075157 & 4075166 & + & TTANCGNTAA & TTAACGATAA & dnaE & gene & 4075157 & 4078678 & - \\
\hline Pseudomonas aeruginosa $\mathrm{PAO} 1$ & 4075157 & 4075166 & - & TTANCGNTAA & TTATCGTTAA & dnaE & gene & 4075157 & 4078678 & - \\
\hline Pseudomonas aeruginosa PAO1 & 4288882 & 4288891 & + & TTANCGNTAA & TTACCGCTAA & & intergenic & & & \\
\hline Pseudomonas aeruginosa PAO1 & 4288882 & 4288891 & - & TTANCGNTAA & TTAGCGGTAA & & intergenic & & & \\
\hline Pseudomonas aeruginosa PAO1 & 5242860 & 5242869 & + & TTANCGNTAA & TTAACGTTAA & & intergenic & & & \\
\hline Pseudomonas aeruginosa PAO1 & 5242860 & 5242869 & - & TTANCGNTAA & TTAACGTTAA & & intergenic & & & \\
\hline Pseudomonas aeruginosa PAO1 & 355178 & 355187 & + & TTANCGTNAA & TTACCGTCAA & PA0315 & gene & 354754 & 355191 & + \\
\hline Pseudomonas aeruginosa PAO1 & 653774 & 653783 & - & TTANCGTNAA & TTATCGTCAA & lptD & gene & 653753 & 656527 & - \\
\hline Pseudomonas aeruginosa PAO1 & 674009 & 674018 & - & TTANCGTNAA & TTATCGTCAA & & intergenic & & & \\
\hline Pseudomonas aeruginosa PAO1 & 786187 & 786196 & + & TTANCGTNAA & TTATCGTAAA & PA0715 & gene & 785969 & 786925 & + \\
\hline Pseudomonas aeruginosa PAO1 & 991605 & 991614 & - & TTANCGTNAA & TTACCGTGAA & & intergenic & & & \\
\hline Pseudomonas aeruginosa PAO1 & 1087474 & 1087483 & - & TTANCGTNAA & TTAACGTTAA & & intergenic & & & \\
\hline Pseudomonas aeruginosa PAO1 & 1087474 & 1087483 & + & TTANCGTNAA & TTAACGTTAA & & intergenic & & & \\
\hline Pseudomonas aeruginosa PAO1 & 1397213 & 1397222 & - & TTANCGTNAA & TTATCGTGAA & & intergenic & & & \\
\hline
\end{tabular}




\begin{tabular}{|c|c|c|c|c|c|c|c|c|c|c|}
\hline Pseudomonas aeruginosa PAO1 & 1618304 & 1618313 & + & TTANCGTNAA & TTACCGTGAA & & intergenic & & & \\
\hline Pseudomonas aeruginosa $\mathrm{PAO} 1$ & 1953368 & 1953377 & + & TTANCGTNAA & TTACCGTCAA & tig & gene & 1952665 & 1953975 & + \\
\hline Pseudomonas aeruginosa PAO1 & 2206830 & 2206839 & - & TTANCGTNAA & TTAACGTAAA & & intergenic & & & \\
\hline Pseudomonas aeruginosa $\mathrm{PAO1}$ & 2206836 & 2206845 & + & TTANCGTNAA & TTAACGTAAA & & intergenic & & & \\
\hline Pseudomonas aeruginosa PAO1 & 2892161 & 2892170 & - & TTANCGTNAA & TTAACGTAAA & & intergenic & & & \\
\hline Pseudomonas aeruginosa PAO1 & 2892167 & 2892176 & + & TTANCGTNAA & TTAACGTAAA & & intergenic & & & \\
\hline Pseudomonas aeruginosa $\mathrm{PAO} 1$ & 2979487 & 2979496 & + & TTANCGTNAA & TTAGCGTCAA & & intergenic & & & \\
\hline Pseudomonas aeruginosa PAO1 & 3097475 & 3097484 & - & TTANCGTNAA & TTATCGTCAA & PA2735 & gene & 3096130 & 3098508 & - \\
\hline Pseudomonas aeruginosa PAO1 & 3141634 & 3141643 & - & TTANCGTNAA & TTATCGTAAA & & intergenic & & & \\
\hline Pseudomonas aeruginosa $\mathrm{PAO1}$ & 3156441 & 3156450 & - & TTANCGTNAA & TTATCGTCAA & & intergenic & & & \\
\hline Pseudomonas aeruginosa PAO1 & 3325584 & 3325593 & - & TTANCGTNAA & TTACCGTGAA & fabG & gene & 3325379 & 3326122 & - \\
\hline Pseudomonas aeruginosa PAO1 & 3434013 & 3434022 & + & TTANCGTNAA & TTAACGTCAA & & intergenic & & & \\
\hline Pseudomonas aeruginosa PAO1 & 3530112 & 3530121 & + & TTANCGTNAA & TTACCGTTAA & wbpK & gene & 3529508 & 3530458 & - \\
\hline Pseudomonas aeruginosa $\mathrm{PAO} 1$ & 3542936 & 3542945 & - & TTANCGTNAA & TTACCGTCAA & wbpB & gene & 3542740 & 3543690 & - \\
\hline Pseudomonas aeruginosa PAO1 & 3610559 & 3610568 & - & TTANCGTNAA & TTACCGTGAA & PA3222 & gene & 3610250 & 3611149 & + \\
\hline Pseudomonas aeruginosa PAO1 & 4075157 & 4075166 & - & TTANCGTNAA & TTATCGTTAA & dnaE & gene & 4075157 & 4078678 & - \\
\hline Pseudomonas aeruginosa PAO1 & 4626505 & 4626514 & + & TTANCGTNAA & TTACCGTCAA & & intergenic & & & \\
\hline Pseudomonas aeruginosa PAO1 & 4925851 & 4925860 & - & TTANCGTNAA & TTACCGTCAA & & intergenic & & & \\
\hline Pseudomonas aeruginosa PAO1 & 4974135 & 4974144 & + & TTANCGTNAA & TTAACGTAAA & & intergenic & & & \\
\hline Pseudomonas aeruginosa PAO1 & 5131447 & 5131456 & + & TTANCGTNAA & TTATCGTGAA & PA4582 & gene & 5131428 & 5132573 & + \\
\hline Pseudomonas aeruginosa PAO1 & 5242860 & 5242869 & + & TTANCGTNAA & TTAACGTTAA & & intergenic & & & \\
\hline Pseudomonas aeruginosa PAO1 & 5242860 & 5242869 & - & TTANCGTNAA & TTAACGTTAA & & intergenic & & & \\
\hline Pseudomonas aeruginosa PAO1 & 5923502 & 5923511 & - & TTANCGTNAA & TTATCGTGAA & fims & gene & 5923295 & 5924371 & - \\
\hline Pseudomonas aeruginosa PAO1 & 123740 & 123749 & + & TTANCGTTNA & TTAGCGTTGA & & intergenic & & & \\
\hline Pseudomonas aeruginosa PAO1 & 1069111 & 1069120 & - & TTANCGTTNA & TTACCGTTGA & PA0987 & gene & 1068489 & 1069331 & + \\
\hline Pseudomonas aeruginosa PAO1 & 1087474 & 1087483 & + & TTANCGTTNA & TTAACGTTAA & & intergenic & & & \\
\hline Pseudomonas aeruginosa PAO1 & 1087474 & 1087483 & - & TTANCGTTNA & TTAACGTTAA & & intergenic & & & \\
\hline Pseudomonas aeruginosa PAO1 & 1467867 & 1467876 & + & TTANCGTTNA & TTACCGTTGA & PA1353 & gene & 1467488 & 1467901 & - \\
\hline Pseudomonas aeruginosa PAO1 & 3120854 & 3120863 & - & TTANCGTTNA & TTACCGTTGA & oprQ & gene & 3120073 & 3121350 & + \\
\hline Pseudomonas aeruginosa $\mathrm{PAO} 1$ & 3530112 & 3530121 & + & TTANCGTTNA & TTACCGTTAA & wbpK & gene & 3529508 & 3530458 & - \\
\hline Pseudomonas aeruginosa PAO1 & 4075157 & 4075166 & - & TTANCGTTNA & TTATCGTTAA & dnaE & gene & 4075157 & 4078678 & - \\
\hline Pseudomonas aeruginosa PAO1 & 5120078 & 5120087 & - & TTANCGTTNA & TTACCGTTCA & PA4571 & gene & 5118538 & 5120565 & + \\
\hline Pseudomonas aeruginosa PAO1 & 5242860 & 5242869 & - & TTANCGTTNA & TTAACGTTAA & & intergenic & & & \\
\hline Pseudomonas aeruginosa PAO1 & 5242860 & 5242869 & + & TTANCGTTNA & TTAACGTTAA & & intergenic & & & \\
\hline Pseudomonas aeruginosa PAO1 & 5680727 & 5680736 & + & TTANCGTTNA & TTAGCGTTCA & & intergenic & & & \\
\hline Pseudomonas aeruginosa PAO1 & 225242 & 225251 & - & TTANCGTTAN & TTATCGTTAT & & intergenic & & & \\
\hline Pseudomonas aeruginosa PAO1 & 1087474 & 1087483 & + & TTANCGTTAN & TTAACGTTAA & & intergenic & & & \\
\hline Pseudomonas aeruginosa PAO1 & 1087474 & 1087483 & - & TTANCGTTAN & TTAACGTTAA & & intergenic & & & \\
\hline Pseudomonas aeruginosa PAO1 & 1117641 & 1117650 & - & TTANCGTTAN & TTATCGTTAT & & intergenic & & & \\
\hline Pseudomonas aeruginosa PAO1 & 1665090 & 1665099 & + & TTANCGTTAN & TTATCGTTAT & zipA & gene & 1665065 & 1665934 & + \\
\hline Pseudomonas aeruginosa PAO1 & 1666090 & 1666099 & + & TTANCGTTAN & TTACCGTTAC & lig & gene & 1666025 & 1668409 & + \\
\hline Pseudomonas aeruginosa PAO1 & 1723297 & 1723306 & + & TTANCGTTAN & TTATCGTTAC & $\operatorname{sdh} B$ & gene & 1723280 & 1723987 & + \\
\hline Pseudomonas aeruginosa PAO1 & 2544919 & 2544928 & - & TTANCGTTAN & TTACCGTTAC & $\mathrm{ambB}$ & gene & 2541197 & 2544946 & - \\
\hline
\end{tabular}




\begin{tabular}{|c|c|c|c|c|c|c|c|c|c|c|}
\hline Pseudomonas aeruginosa PAO1 & 2556300 & 2556309 & + & TTANCGTTAN & TTACCGTTAT & PA2318 & gene & 2556293 & 2556658 & + \\
\hline Pseudomonas aeruginosa PAO1 & 3483666 & 3483675 & - & TTANCGTTAN & TTATCGTTAT & хсрP & gene & 3483642 & 3484349 & + \\
\hline Pseudomonas aeruginosa PAO1 & 3530112 & 3530121 & + & TTANCGTTAN & TTACCGTTAA & wbpK & gene & 3529508 & 3530458 & - \\
\hline Pseudomonas aeruginosa PAO1 & 3683919 & 3683928 & + & TTANCGTTAN & TTATCGTTAT & tle1 & gene & 3681500 & 3684148 & - \\
\hline Pseudomonas aeruginosa PAO1 & 3893261 & 3893270 & - & TTANCGTTAN & TTACCGTTAG & & intergenic & & & \\
\hline Pseudomonas aeruginosa PAO1 & 4033928 & 4033937 & - & TTANCGTTAN & TTACCGTTAC & PA3598 & gene & 4033850 & 4034665 & - \\
\hline Pseudomonas aeruginosa $\mathrm{PAO} 1$ & 4075157 & 4075166 & - & TTANCGTTAN & TTATCGTTAA & dnaE & gene & 4075157 & 4078678 & - \\
\hline Pseudomonas aeruginosa PAO1 & 4609488 & 4609497 & - & TTANCGTTAN & TTAACGTTAT & & intergenic & & & \\
\hline Pseudomonas aeruginosa PAO1 & 4663708 & 4663717 & - & TTANCGTTAN & TTATCGTTAT & & intergenic & & & \\
\hline Pseudomonas aeruginosa $\mathrm{PAO1}$ & 5242860 & 5242869 & + & TTANCGTTAN & TTAACGTTAA & & intergenic & & & \\
\hline Pseudomonas aeruginosa PAO1 & 5242860 & 5242869 & - & TTANCGTTAN & TTAACGTTAA & & intergenic & & & \\
\hline Pseudomonas aeruginosa PAO1 & 5786723 & 5786732 & + & TTANCGTTAN & TTATCGTTAT & & intergenic & & & \\
\hline Pseudomonas aeruginosa PAO1 & 355178 & 355187 & - & TTNACGNTAA & TTGACGGTAA & PA0315 & gene & 354754 & 355191 & + \\
\hline Pseudomonas aeruginosa PAO1 & 653774 & 653783 & + & TTNACGNTAA & TTGACGATAA & lptD & gene & 653753 & 656527 & - \\
\hline Pseudomonas aeruginosa PAO1 & 674009 & 674018 & + & TTNACGNTAA & TTGACGATAA & & intergenic & & & \\
\hline Pseudomonas aeruginosa PAO1 & 786187 & 786196 & - & TTNACGNTAA & TTTACGATAA & PA0715 & gene & 785969 & 786925 & + \\
\hline Pseudomonas aeruginosa PAO1 & 991605 & 991614 & + & TTNACGNTAA & TTCACGGTAA & & intergenic & & & \\
\hline Pseudomonas aeruginosa PAO1 & 1087474 & 1087483 & + & TTNACGNTAA & TTAACGTTAA & & intergenic & & & \\
\hline Pseudomonas aeruginosa PAO1 & 1087474 & 1087483 & - & TTNACGNTAA & TTAACGTTAA & & intergenic & & & \\
\hline Pseudomonas aeruginosa PAO1 & 1397213 & 1397222 & + & TTNACGNTAA & TTCACGATAA & & intergenic & & & \\
\hline Pseudomonas aeruginosa PAO1 & 1618304 & 1618313 & - & TTNACGNTAA & TTCACGGTAA & & intergenic & & & \\
\hline Pseudomonas aeruginosa PAO1 & 1953368 & 1953377 & - & TTNACGNTAA & TTGACGGTAA & tig & gene & 1952665 & 1953975 & + \\
\hline Pseudomonas aeruginosa $\mathrm{PAO} 1$ & 2206830 & 2206839 & + & TTNACGNTAA & TTTACGTTAA & & intergenic & & & \\
\hline Pseudomonas aeruginosa PAO1 & 2206836 & 2206845 & - & TTNACGNTAA & TTTACGTTAA & & intergenic & & & \\
\hline Pseudomonas aeruginosa PAO1 & 2892161 & 2892170 & + & TTNACGNTAA & TTTACGTTAA & & intergenic & & & \\
\hline Pseudomonas aeruginosa PAO1 & 2892167 & 2892176 & - & TTNACGNTAA & TTTACGTTAA & & intergenic & & & \\
\hline Pseudomonas aeruginosa PAO1 & 2979487 & 2979496 & - & TTNACGNTAA & TTGACGCTAA & & intergenic & & & \\
\hline Pseudomonas aeruginosa PAO1 & 3097475 & 3097484 & + & TTNACGNTAA & TTGACGATAA & PA2735 & gene & 3096130 & 3098508 & - \\
\hline Pseudomonas aeruginosa PAO1 & 3141634 & 3141643 & + & TTNACGNTAA & TTTACGATAA & & intergenic & & & \\
\hline Pseudomonas aeruginosa PAO1 & 3156441 & 3156450 & + & TTNACGNTAA & TTGACGATAA & & intergenic & & & \\
\hline Pseudomonas aeruginosa PAO1 & 3325584 & 3325593 & + & TTNACGNTAA & TTCACGGTAA & fabG & gene & 3325379 & 3326122 & - \\
\hline Pseudomonas aeruginosa PAO1 & 3434013 & 3434022 & - & TTNACGNTAA & TTGACGTTAA & & intergenic & & & \\
\hline Pseudomonas aeruginosa PAO1 & 3530112 & 3530121 & - & TTNACGNTAA & TTAACGGTAA & wbpK & gene & 3529508 & 3530458 & - \\
\hline Pseudomonas aeruginosa PAO1 & 3542936 & 3542945 & + & TTNACGNTAA & TTGACGGTAA & wbpB & gene & 3542740 & 3543690 & - \\
\hline Pseudomonas aeruginosa PAO1 & 3610559 & 3610568 & + & TTNACGNTAA & TTCACGGTAA & PA3222 & gene & 3610250 & 3611149 & + \\
\hline Pseudomonas aeruginosa PAO1 & 4075157 & 4075166 & + & TTNACGNTAA & TTAACGATAA & dnaE & gene & 4075157 & 4078678 & - \\
\hline Pseudomonas aeruginosa PAO1 & 4626505 & 4626514 & - & TTNACGNTAA & TTGACGGTAA & & intergenic & & & \\
\hline Pseudomonas aeruginosa PAO1 & 4925851 & 4925860 & + & TTNACGNTAA & TTGACGGTAA & & intergenic & & & \\
\hline Pseudomonas aeruginosa PAO1 & 4974135 & 4974144 & - & TTNACGNTAA & TTTACGTTAA & & intergenic & & & \\
\hline Pseudomonas aeruginosa PAO1 & 5131447 & 5131456 & - & TTNACGNTAA & TTCACGATAA & PA4582 & gene & 5131428 & 5132573 & + \\
\hline Pseudomonas aeruginosa PAO1 & 5242860 & 5242869 & + & TTNACGNTAA & TTAACGTTAA & & intergenic & & & \\
\hline Pseudomonas aeruginosa PAO1 & 5242860 & 5242869 & - & TTNACGNTAA & TTAACGTTAA & & intergenic & & & \\
\hline Pseudomonas aeruginosa PAO1 & 5923502 & 5923511 & + & TTNACGNTAA & TTCACGATAA & fims & gene & 5923295 & 5924371 & - \\
\hline
\end{tabular}




\begin{tabular}{|c|c|c|c|c|c|c|c|c|c|c|}
\hline Pseudomonas aeruginosa PAO1 & 1087474 & 1087483 & + & TTNACGTNAA & TTAACGTTAA & & intergenic & & & \\
\hline Pseudomonas aeruginosa PAO1 & 1087474 & 1087483 & - & TTNACGTNAA & TTAACGTTAA & & intergenic & & & \\
\hline Pseudomonas aeruginosa PAO1 & 2206830 & 2206839 & + & TTNACGTNAA & TTTACGTTAA & & intergenic & & & \\
\hline Pseudomonas aeruginosa $\mathrm{PAO1}$ & 2206830 & 2206839 & - & TTNACGTNAA & TTAACGTAAA & & intergenic & & & \\
\hline Pseudomonas aeruginosa PAO1 & 2206836 & 2206845 & + & TTNACGTNAA & TTAACGTAAA & & intergenic & & & \\
\hline Pseudomonas aeruginosa PAO1 & 2206836 & 2206845 & - & TTNACGTNAA & TTTACGTTAA & & intergenic & & & \\
\hline Pseudomonas aeruginosa $\mathrm{PAO} 1$ & 2892161 & 2892170 & + & TTNACGTNAA & TTTACGTTAA & & intergenic & & & \\
\hline Pseudomonas aeruginosa PAO1 & 2892161 & 2892170 & - & TTNACGTNAA & TTAACGTAAA & & intergenic & & & \\
\hline Pseudomonas aeruginosa PAO1 & 2892167 & 2892176 & + & TTNACGTNAA & TTAACGTAAA & & intergenic & & & \\
\hline Pseudomonas aeruginosa $\mathrm{PAO1}$ & 2892167 & 2892176 & - & TTNACGTNAA & TTTACGTTAA & & intergenic & & & \\
\hline Pseudomonas aeruginosa PAO1 & 3434013 & 3434022 & + & TTNACGTNAA & TTAACGTCAA & & intergenic & & & \\
\hline Pseudomonas aeruginosa PAO1 & 3434013 & 3434022 & - & TTNACGTNAA & TTGACGTTAA & & intergenic & & & \\
\hline Pseudomonas aeruginosa PAO1 & 3791221 & 3791230 & + & TTNACGTNAA & TTGACGTCAA & & intergenic & & & \\
\hline Pseudomonas aeruginosa PAO1 & 3791221 & 3791230 & - & TTNACGTNAA & TTGACGTCAA & & intergenic & & & \\
\hline Pseudomonas aeruginosa PAO1 & 4974135 & 4974144 & + & TTNACGTNAA & TTAACGTAAA & & intergenic & & & \\
\hline Pseudomonas aeruginosa PAO1 & 4974135 & 4974144 & - & TTNACGTNAA & TTTACGTTAA & & intergenic & & & \\
\hline Pseudomonas aeruginosa PAO1 & 5242860 & 5242869 & + & TTNACGTNAA & TTAACGTTAA & & intergenic & & & \\
\hline Pseudomonas aeruginosa PAO1 & 5242860 & 5242869 & - & TTNACGTNAA & TTAACGTTAA & & intergenic & & & \\
\hline Pseudomonas aeruginosa PAO1 & 117466 & 117475 & - & TTNACGTTNA & TTGACGTTGA & PA0095 & gene & 115299 & 117524 & + \\
\hline Pseudomonas aeruginosa PAO1 & 120489 & 120498 & - & TTNACGTTNA & TTGACGTTCA & PA0099 & gene & 120164 & 121324 & + \\
\hline Pseudomonas aeruginosa PAO1 & 173322 & 173331 & - & TTNACGTTNA & TTCACGTTGA & PA0151 & gene & 171047 & 173434 & + \\
\hline Pseudomonas aeruginosa PAO1 & 196267 & 196276 & + & TTNACGTTNA & TTGACGTTGA & $\operatorname{siaA}$ & gene & 194757 & 196748 & - \\
\hline Pseudomonas aeruginosa PAO1 & 230438 & 230447 & - & TTNACGTTNA & TTCACGTTGA & PA0201 & gene & 229954 & 230535 & + \\
\hline Pseudomonas aeruginosa PAO1 & 283036 & 283045 & + & TTNACGTTNA & TTGACGTTCA & PA0251 & gene & 282912 & 283553 & - \\
\hline Pseudomonas aeruginosa PAO1 & 350966 & 350975 & - & TTNACGTTNA & TTGACGTTGA & PA0310 & gene & 350890 & 351588 & + \\
\hline Pseudomonas aeruginosa PAO1 & 439392 & 439401 & + & TTNACGTTNA & TTGACGTTCA & PA0397 & gene & 438938 & 439837 & - \\
\hline Pseudomonas aeruginosa PAO1 & 483550 & 483559 & + & TTNACGTTNA & TTGACGTTGA & sahH & gene & 482706 & 484115 & - \\
\hline Pseudomonas aeruginosa PAO1 & 579130 & 579139 & + & TTNACGTTNA & TTCACGTTGA & nirS & gene & 578394 & 580100 & - \\
\hline Pseudomonas aeruginosa PAO1 & 613405 & 613414 & - & TTNACGTTNA & TTGACGTTGA & fda & gene & 613338 & 614402 & + \\
\hline Pseudomonas aeruginosa PAO1 & 653768 & 653777 & + & TTNACGTTNA & TTCACGTTGA & surA & gene & 652480 & 653772 & - \\
\hline Pseudomonas aeruginosa PAO1 & 669953 & 669962 & - & TTNACGTTNA & TTGACGTTGA & rpe & gene & 669415 & 670089 & + \\
\hline Pseudomonas aeruginosa PAO1 & 933799 & 933808 & + & TTNACGTTNA & TTGACGTTCA & fumC2 & gene & 932718 & 934112 & - \\
\hline Pseudomonas aeruginosa PAO1 & 953767 & 953776 & - & TTNACGTTNA & TTGACGTTGA & phhR & gene & 953691 & 955250 & + \\
\hline Pseudomonas aeruginosa PAO1 & 1056995 & 1057004 & - & TTNACGTTNA & TTCACGTTCA & tolB & gene & 1056049 & 1057347 & + \\
\hline Pseudomonas aeruginosa PAO1 & 1087474 & 1087483 & - & TTNACGTTNA & TTAACGTTAA & & intergenic & & & \\
\hline Pseudomonas aeruginosa PAO1 & 1087474 & 1087483 & + & TTNACGTTNA & TTAACGTTAA & & intergenic & & & \\
\hline Pseudomonas aeruginosa PAO1 & 1365985 & 1365994 & + & TTNACGTTNA & TTGACGTTGA & IhpM & gene & 1365873 & 1366538 & - \\
\hline Pseudomonas aeruginosa PAO1 & 1583477 & 1583486 & - & TTNACGTTNA & TTCACGTTCA & flhF & gene & 1582528 & 1583817 & + \\
\hline Pseudomonas aeruginosa PAO1 & 1588926 & 1588935 & - & TTNACGTTNA & TTGACGTTGA & PA1458 & gene & 1587023 & 1589284 & + \\
\hline Pseudomonas aeruginosa PAO1 & 1590050 & 1590059 & - & TTNACGTTNA & TTGACGTTGA & PA1459 & gene & 1589338 & 1590444 & + \\
\hline Pseudomonas aeruginosa PAO1 & 1751006 & 1751015 & + & TTNACGTTNA & TTGACGTTGA & PA1608 & gene & 1749895 & 1751520 & - \\
\hline Pseudomonas aeruginosa PAO1 & 1796481 & 1796490 & - & TTNACGTTNA & TTCACGTTGA & PA1649 & gene & 1796138 & 1796899 & + \\
\hline Pseudomonas aeruginosa PAO1 & 1857018 & 1857028 & - & TTNACGTTNA & TTCACGTTCA & & intergenic & & & \\
\hline
\end{tabular}




\begin{tabular}{|c|c|c|c|c|c|c|c|c|c|c|}
\hline Pseudomonas aeruginosa PAO1 & 1880016 & 1880025 & - & TTNACGTTNA & TTGACGTTGA & PA1736 & gene & 1878980 & 1880185 & + \\
\hline Pseudomonas aeruginosa $\mathrm{PAO} 1$ & 1906725 & 1906734 & - & TTNACGTTNA & TTGACGTTGA & PA1763 & gene & 1905797 & 1906822 & + \\
\hline Pseudomonas aeruginosa PAO1 & 2120385 & 2120394 & + & TTNACGTTNA & TTCACGTTGA & PA1939 & gene & 2120225 & 2122222 & - \\
\hline Pseudomonas aeruginosa $\mathrm{PAO1}$ & 2188077 & 2188086 & - & TTNACGTTNA & TTGACGTTGA & atoB & gene & 2187065 & 2188246 & + \\
\hline Pseudomonas aeruginosa PAO1 & 2206830 & 2206839 & + & TTNACGTTNA & TTTACGTTAA & & intergenic & & & \\
\hline Pseudomonas aeruginosa $\mathrm{PAO} 1$ & 2206836 & 2206845 & - & TTNACGTTNA & TTTACGTTAA & & intergenic & & & \\
\hline Pseudomonas aeruginosa $\mathrm{PAO} 1$ & 2412557 & 2412566 & - & TTNACGTTNA & TTGACGTTCA & hcnA & gene & 2412546 & 2412860 & + \\
\hline Pseudomonas aeruginosa PAO1 & 2563855 & 2563864 & - & TTNACGTTNA & TTGACGTTGA & gapN & gene & 2562447 & 2564072 & + \\
\hline Pseudomonas aeruginosa PAO1 & 2625663 & 2625672 & - & TTNACGTTNA & TTGACGTTGA & vgrG3 & gene & 2624204 & 2626210 & + \\
\hline Pseudomonas aeruginosa PAO1 & 2657522 & 2657531 & - & TTNACGTTNA & TTGACGTTGA & fpvA & gene & 2655187 & 2657634 & + \\
\hline Pseudomonas aeruginosa PAO1 & 2756845 & 2756854 & - & TTNACGTTNA & TTCACGTTGA & & intergenic & & & \\
\hline Pseudomonas aeruginosa PAO1 & 2810493 & 2810502 & - & TTNACGTTNA & TTCACGTTGA & $\operatorname{mexF}$ & gene & 2810009 & 2813197 & + \\
\hline Pseudomonas aeruginosa PAO1 & 2855014 & 2855023 & + & TTNACGTTNA & TTGACGTTCA & $\operatorname{muxA}$ & gene & 2854011 & 2855291 & - \\
\hline Pseudomonas aeruginosa PAO1 & 2874311 & 2874320 & + & TTNACGTTNA & TTGACGTTGA & PA2543 & gene & 2873824 & 2875563 & - \\
\hline Pseudomonas aeruginosa $\mathrm{PAO} 1$ & 2892161 & 2892170 & + & TTNACGTTNA & TTTACGTTAA & & intergenic & & & \\
\hline Pseudomonas aeruginosa PAO1 & 2892167 & 2892176 & - & TTNACGTTNA & TTTACGTTAA & & intergenic & & & \\
\hline Pseudomonas aeruginosa PAO1 & 2933649 & 2933658 & + & TTNACGTTNA & TTCACGTTCA & vqsR & gene & 2933582 & 2934388 & - \\
\hline Pseudomonas aeruginosa PAO1 & 2991965 & 2991974 & - & TTNACGTTNA & TTCACGTTGA & nuoI & gene & 2991442 & 2991990 & + \\
\hline Pseudomonas aeruginosa PAO1 & 3320689 & 3320698 & + & TTNACGTTNA & TTCACGTTCA & holB & gene & 3320064 & 3321050 & - \\
\hline Pseudomonas aeruginosa PAO1 & 3431832 & 3431841 & + & TTNACGTTNA & TTGACGTTCA & pelA & gene & 3431045 & 3433891 & - \\
\hline Pseudomonas aeruginosa $\mathrm{PAO} 1$ & 3434013 & 3434022 & - & TTNACGTTNA & TTGACGTTAA & & intergenic & & & \\
\hline Pseudomonas aeruginosa $\mathrm{PAO} 1$ & 3477970 & 3477979 & + & TTNACGTTNA & TTCACGTTCA & $\mathrm{xcpX}$ & gene & 3477627 & 3478628 & - \\
\hline Pseudomonas aeruginosa PAO1 & 3563916 & 3563925 & - & TTNACGTTNA & TTGACGTTCA & PA3173 & gene & 3563546 & 3564286 & + \\
\hline Pseudomonas aeruginosa PAO1 & 3570139 & 3570148 & + & TTNACGTTNA & TTGACGTTGA & PA3179 & gene & 3569106 & 3570266 & - \\
\hline Pseudomonas aeruginosa PAO1 & 3762526 & 3762535 & - & TTNACGTTNA & TTGACGTTGA & PA3350 & gene & 3761961 & 3762659 & + \\
\hline Pseudomonas aeruginosa PAO1 & 3804696 & 3804705 & + & TTNACGTTNA & TTCACGTTGA & PA3399 & gene & 3804545 & 3804886 & - \\
\hline Pseudomonas aeruginosa $\mathrm{PAO} 1$ & 3944861 & 3944870 & + & TTNACGTTNA & TTCACGTTGA & $\operatorname{argG}$ & gene & 3944662 & 3945879 & - \\
\hline Pseudomonas aeruginosa PAO1 & 3965855 & 3965864 & - & TTNACGTTNA & TTGACGTTGA & alg44 & gene & 3965842 & 3967011 & + \\
\hline Pseudomonas aeruginosa $\mathrm{PAO} 1$ & 3973243 & 3973252 & - & TTNACGTTNA & TTGACGTTGA & algL & gene & 3973014 & 3974117 & + \\
\hline Pseudomonas aeruginosa PAO1 & 4060896 & 4060905 & + & TTNACGTTNA & TTGACGTTGA & surE & gene & 4060625 & 4061374 & - \\
\hline Pseudomonas aeruginosa $\mathrm{PAO} 1$ & 4062511 & 4062520 & + & TTNACGTTNA & TTGACGTTGA & ygbB & gene & 4062426 & 4062899 & - \\
\hline Pseudomonas aeruginosa PAO1 & 4215252 & 4215261 & + & TTNACGTTNA & TTTACGTTCA & & intergenic & & & \\
\hline Pseudomonas aeruginosa PAO1 & 4298546 & 4298555 & - & TTNACGTTNA & TTGACGTTGA & PA3837 & gene & 4298311 & 4299201 & + \\
\hline Pseudomonas aeruginosa PAO1 & 4560306 & 4560315 & - & TTNACGTTNA & TTCACGTTTA & cupB6 & gene & 4560295 & 4561440 & - \\
\hline Pseudomonas aeruginosa PAO1 & 4560674 & 4560683 & + & TTNACGTTNA & TTGACGTTGA & сupB6 & gene & 4560295 & 4561440 & - \\
\hline Pseudomonas aeruginosa PAO1 & 4642063 & 4642072 & - & TTNACGTTNA & TTGACGTTCA & PA4148 & gene & 4641711 & 4642511 & + \\
\hline Pseudomonas aeruginosa PAO1 & 4668515 & 4668524 & - & TTNACGTTNA & TTGACGTTGA & PA4172 & gene & 4668490 & 4669290 & + \\
\hline Pseudomonas aeruginosa PAO1 & 4702892 & 4702901 & + & TTNACGTTNA & TTGACGTTGA & nmoA & gene & 4702042 & 4703097 & - \\
\hline Pseudomonas aeruginosa PAO1 & 4724745 & 4724754 & + & TTNACGTTNA & TTGACGTTGA & fptA & gene & 4724639 & 4726801 & - \\
\hline Pseudomonas aeruginosa PAO1 & 4750953 & 4750962 & - & TTNACGTTNA & TTGACGTTGA & uvrA & gene & 4748756 & 4751593 & + \\
\hline Pseudomonas aeruginosa PAO1 & 4756214 & 4756223 & + & TTNACGTTNA & TTGACGTTGA & rpsK & gene & 4756084 & 4756473 & - \\
\hline Pseudomonas aeruginosa PAO1 & 4765825 & 4765834 & + & TTNACGTTNA & TTGACGTTCA & $\mathrm{rplW}$ & gene & 4765713 & 4766012 & - \\
\hline Pseudomonas aeruginosa PAO1 & 4787808 & 4787817 & + & TTNACGTTNA & TTGACGTTGA & birA & gene & 4787477 & 4788415 & - \\
\hline
\end{tabular}




\begin{tabular}{|c|c|c|c|c|c|c|c|c|c|c|}
\hline Pseudomonas aeruginosa PAO1 & 4815299 & 4815308 & - & TTNACGTTNA & TTCACGTTGA & PA4292 & gene & 4815043 & 4816512 & + \\
\hline Pseudomonas aeruginosa PAO1 & 4821593 & 4821602 & + & TTNACGTTNA & TTGACGTTGA & $\operatorname{tadG}$ & gene & 4821385 & 4823055 & - \\
\hline Pseudomonas aeruginosa PAO1 & 4821680 & 4821689 & + & TTNACGTTNA & TTCACGTTGA & $\operatorname{tadG}$ & gene & 4821385 & 4823055 & - \\
\hline Pseudomonas aeruginosa PAO1 & 4974135 & 4974144 & - & TTNACGTTNA & TTTACGTTAA & & intergenic & & & \\
\hline Pseudomonas aeruginosa PAO1 & 5070862 & 5070871 & - & TTNACGTTNA & TTGACGTTGA & pilB & gene & 5069763 & 5071463 & + \\
\hline Pseudomonas aeruginosa $\mathrm{PAO1}$ & 5085486 & 5085495 & - & TTNACGTTNA & TTTACGTTGA & lepA & gene & 5082443 & 5086696 & + \\
\hline Pseudomonas aeruginosa $\mathrm{PAO} 1$ & 5242860 & 5242869 & - & TTNACGTTNA & TTAACGTTAA & & intergenic & & & \\
\hline Pseudomonas aeruginosa PAO1 & 5242860 & 5242869 & + & TTNACGTTNA & TTAACGTTAA & & intergenic & & & \\
\hline Pseudomonas aeruginosa PAO1 & 5348216 & 5348225 & + & TTNACGTTNA & TTGACGTTCA & dnaK & gene & 5347198 & 5349111 & - \\
\hline Pseudomonas aeruginosa $\mathrm{PAO1}$ & 5372748 & 5372757 & + & TTNACGTTNA & TTGACGTTGA & PA4785 & gene & 5372591 & 5373868 & - \\
\hline Pseudomonas aeruginosa PAO1 & 5375060 & 5375069 & - & TTNACGTTNA & TTGACGTTGA & PA4786 & gene & 5374123 & 5375478 & + \\
\hline Pseudomonas aeruginosa PAO1 & 5427819 & 5427828 & + & TTNACGTTNA & TTGACGTTCA & cntO & gene & 5427716 & 5429842 & - \\
\hline Pseudomonas aeruginosa PAO1 & 5623827 & 5623836 & + & TTNACGTTNA & TTGACGTTCA & PA5005 & gene & 5623040 & 5624797 & - \\
\hline Pseudomonas aeruginosa PAO1 & 5799087 & 5799096 & + & TTNACGTTNA & TTGACGTTGA & PA5 150 & gene & 5798693 & 5799430 & - \\
\hline Pseudomonas aeruginosa PAO1 & 5974266 & 5974275 & + & TTNACGTTNA & TTCACGTTGA & PA5306 & gene & 5974190 & 5974381 & - \\
\hline Pseudomonas aeruginosa PAO1 & 5974520 & 5974529 & + & TTNACGTTNA & TTGACGTTGA & PA5307 & gene & 5974402 & 5976969 & - \\
\hline Pseudomonas aeruginosa PAO1 & 6002137 & 6002146 & - & TTNACGTTNA & TTCACGTTCA & crc & gene & 6002121 & 6002900 & + \\
\hline Pseudomonas aeruginosa PAO1 & 722568 & 722577 & + & TTNACGTTAN & TTGACGTTAC & PA0668.1 & gene & 722096 & 723631 & + \\
\hline Pseudomonas aeruginosa PAO1 & 1060321 & 1060330 & + & TTNACGTTAN & TTTACGTTAG & & intergenic & & & \\
\hline Pseudomonas aeruginosa PAO1 & 1087474 & 1087483 & + & TTNACGTTAN & TTAACGTTAA & & intergenic & & & \\
\hline Pseudomonas aeruginosa PAO1 & 1087474 & 1087483 & - & TTNACGTTAN & TTAACGTTAA & & intergenic & & & \\
\hline Pseudomonas aeruginosa PAO1 & 2206830 & 2206839 & + & TTNACGTTAN & TTTACGTTAA & & intergenic & & & \\
\hline Pseudomonas aeruginosa $\mathrm{PAO} 1$ & 2206836 & 2206845 & - & TTNACGTTAN & TTTACGTTAA & & intergenic & & & \\
\hline Pseudomonas aeruginosa PAO1 & 2892161 & 2892170 & + & TTNACGTTAN & TTTACGTTAA & & intergenic & & & \\
\hline Pseudomonas aeruginosa PAO1 & 2892167 & 2892176 & - & TTNACGTTAN & TTTACGTTAA & & intergenic & & & \\
\hline Pseudomonas aeruginosa PAO1 & 3434013 & 3434022 & - & TTNACGTTAN & TTGACGTTAA & & intergenic & & & \\
\hline Pseudomonas aeruginosa PAO1 & 3751828 & 3751837 & + & TTNACGTTAN & TTCACGTTAG & & intergenic & & & \\
\hline Pseudomonas aeruginosa PAO1 & 4609488 & 4609497 & - & TTNACGTTAN & TTAACGTTAT & & intergenic & & & \\
\hline Pseudomonas aeruginosa PAO1 & 4793251 & 4793260 & - & TTNACGTTAN & TTGACGTTAC & PA4280.5 & gene & 4792196 & 4793731 & - \\
\hline Pseudomonas aeruginosa PAO1 & 4974135 & 4974144 & - & TTNACGTTAN & TTTACGTTAA & & intergenic & & & \\
\hline Pseudomonas aeruginosa PAO1 & 5067823 & 5067832 & - & TTNACGTTAN & TTGACGTTAC & & intergenic & & & \\
\hline Pseudomonas aeruginosa PAO1 & 5242860 & 5242869 & + & TTNACGTTAN & TTAACGTTAA & & intergenic & & & \\
\hline Pseudomonas aeruginosa PAO1 & 5242860 & 5242869 & - & TTNACGTTAN & TTAACGTTAA & & intergenic & & & \\
\hline Pseudomonas aeruginosa PAO1 & 5268779 & 5268788 & - & TTNACGTTAN & TTGACGTTAC & PA4690.5 & gene & 5267724 & 5269259 & - \\
\hline Pseudomonas aeruginosa PAO1 & 5349038 & 5349047 & + & TTNACGTTAN & TTGACGTTAC & dnaK & gene & 5347198 & 5349111 & - \\
\hline Pseudomonas aeruginosa PAO1 & 5926870 & 5926879 & - & TTNACGTTAN & TTCACGTTAT & PA5264 & gene & 5926134 & 5927105 & - \\
\hline Pseudomonas aeruginosa PAO1 & 6044263 & 6044272 & - & TTNACGTTAN & TTGACGTTAC & PA5369.5 & gene & 6043208 & 6044743 & - \\
\hline Pseudomonas aeruginosa PAO1 & 654516 & 654525 & + & TTANCGNTAA & TTACCGGTAA & lptD & gene & 653753 & 656527 & - \\
\hline Pseudomonas aeruginosa PAO1 & 654516 & 654525 & - & TTANCGNTAA & TTACCGGTAA & lptD & gene & 653753 & 656527 & - \\
\hline Pseudomonas aeruginosa PAO1 & 1087474 & 1087483 & + & TTANCGNTAA & TTAACGTTAA & & intergenic & & & \\
\hline Pseudomonas aeruginosa PAO1 & 1087474 & 1087483 & - & TTANCGNTAA & TTAACGTTAA & & intergenic & & & \\
\hline Pseudomonas aeruginosa PAO1 & 1299483 & 1299492 & + & TTANCGNTAA & TTATCGATAA & PA1197 & gene & 1298722 & 1299492 & + \\
\hline Pseudomonas aeruginosa PAO1 & 1299483 & 1299492 & - & TTANCGNTAA & TTATCGATAA & PA1197 & gene & 1298722 & 1299492 & + \\
\hline
\end{tabular}




\begin{tabular}{|c|c|c|c|c|c|c|c|c|c|c|}
\hline Pseudomonas aeruginosa PAO1 & 2120589 & 2120598 & + & TTANCGNTAA & TTATCGATAA & PA1939 & gene & 2120225 & 2122222 & - \\
\hline Pseudomonas aeruginosa PAO1 & 2120589 & 2120598 & - & TTANCGNTAA & TTATCGATAA & PA1939 & gene & 2120225 & 2122222 & - \\
\hline Pseudomonas aeruginosa PAO1 & 3119794 & 3119803 & + & TTANCGNTAA & TTATCGATAA & & intergenic & & & \\
\hline Pseudomonas aeruginosa PAO1 & 3119794 & 3119803 & - & TTANCGNTAA & TTATCGATAA & & intergenic & & & \\
\hline Pseudomonas aeruginosa PAO1 & 3530112 & 3530121 & + & TTANCGNTAA & TTACCGTTAA & wbpK & gene & 3529508 & 3530458 & - \\
\hline Pseudomonas aeruginosa PAO1 & 3530112 & 3530121 & - & TTANCGNTAA & TTAACGGTAA & wbpk & gene & 3529508 & 3530458 & - \\
\hline Pseudomonas aeruginosa $\mathrm{PAO} 1$ & 3937875 & 3937884 & + & TTANCGNTAA & TTATCGATAA & & intergenic & & & \\
\hline Pseudomonas aeruginosa PAO1 & 3937875 & 3937884 & - & TTANCGNTAA & TTATCGATAA & & intergenic & & & \\
\hline Pseudomonas aeruginosa PAO1 & 4075157 & 4075166 & + & TTANCGNTAA & TTAACGATAA & dnaE & gene & 4075157 & 4078678 & - \\
\hline Pseudomonas aeruginosa $\mathrm{PAO1}$ & 4075157 & 4075166 & - & TTANCGNTAA & TTATCGTTAA & dnaE & gene & 4075157 & 4078678 & - \\
\hline Pseudomonas aeruginosa PAO1 & 4288882 & 4288891 & + & TTANCGNTAA & TTACCGCTAA & & intergenic & & & \\
\hline Pseudomonas aeruginosa PAO1 & 4288882 & 4288891 & - & TTANCGNTAA & TTAGCGGTAA & & intergenic & & & \\
\hline Pseudomonas aeruginosa PAO1 & 5242860 & 5242869 & + & TTANCGNTAA & TTAACGTTAA & & intergenic & & & \\
\hline Pseudomonas aeruginosa PAO1 & 5242860 & 5242869 & - & TTANCGNTAA & TTAACGTTAA & & intergenic & & & \\
\hline Pseudomonas aeruginosa PAO1 & 355178 & 355187 & + & TTANCGTNAA & TTACCGTCAA & PA0315 & gene & 354754 & 355191 & + \\
\hline Pseudomonas aeruginosa PAO1 & 653774 & 653783 & - & TTANCGTNAA & TTATCGTCAA & lptD & gene & 653753 & 656527 & - \\
\hline Pseudomonas aeruginosa PAO1 & 674009 & 674018 & - & TTANCGTNAA & TTATCGTCAA & & intergenic & & & \\
\hline Pseudomonas aeruginosa PAO1 & 786187 & 786196 & + & TTANCGTNAA & TTATCGTAAA & PA0715 & gene & 785969 & 786925 & + \\
\hline Pseudomonas aeruginosa PAO1 & 991605 & 991614 & - & TTANCGTNAA & TTACCGTGAA & & intergenic & & & \\
\hline Pseudomonas aeruginosa PAO1 & 1087474 & 1087483 & - & TTANCGTNAA & TTAACGTTAA & & intergenic & & & \\
\hline Pseudomonas aeruginosa PAO1 & 1087474 & 1087483 & + & TTANCGTNAA & TTAACGTTAA & & intergenic & & & \\
\hline Pseudomonas aeruginosa PAO1 & 1397213 & 1397222 & - & TTANCGTNAA & TTATCGTGAA & & intergenic & & & \\
\hline Pseudomonas aeruginosa $\mathrm{PAO} 1$ & 1618304 & 1618313 & + & TTANCGTNAA & TTACCGTGAA & & intergenic & & & \\
\hline Pseudomonas aeruginosa PAO1 & 1953368 & 1953377 & + & TTANCGTNAA & TTACCGTCAA & tig & gene & 1952665 & 1953975 & + \\
\hline Pseudomonas aeruginosa PAO1 & 2206830 & 2206839 & - & TTANCGTNAA & TTAACGTAAA & & intergenic & & & \\
\hline Pseudomonas aeruginosa PAO1 & 2206836 & 2206845 & + & TTANCGTNAA & TTAACGTAAA & & intergenic & & & \\
\hline Pseudomonas aeruginosa PAO1 & 2892161 & 2892170 & - & TTANCGTNAA & TTAACGTAAA & & intergenic & & & \\
\hline Pseudomonas aeruginosa PAO1 & 2892167 & 2892176 & + & TTANCGTNAA & TTAACGTAAA & & intergenic & & & \\
\hline Pseudomonas aeruginosa PAO1 & 2979487 & 2979496 & + & TTANCGTNAA & TTAGCGTCAA & & intergenic & & & \\
\hline Pseudomonas aeruginosa PAO1 & 3097475 & 3097484 & - & TTANCGTNAA & TTATCGTCAA & PA2735 & gene & 3096130 & 3098508 & - \\
\hline Pseudomonas aeruginosa PAO1 & 3141634 & 3141643 & - & TTANCGTNAA & TTATCGTAAA & & intergenic & & & \\
\hline Pseudomonas aeruginosa PAO1 & 3156441 & 3156450 & - & TTANCGTNAA & TTATCGTCAA & & intergenic & & & \\
\hline Pseudomonas aeruginosa PAO1 & 3325584 & 3325593 & - & TTANCGTNAA & TTACCGTGAA & fabG & gene & 3325379 & 3326122 & - \\
\hline Pseudomonas aeruginosa PAO1 & 3434013 & 3434022 & + & TTANCGTNAA & TTAACGTCAA & & intergenic & & & \\
\hline Pseudomonas aeruginosa PAO1 & 3530112 & 3530121 & + & TTANCGTNAA & TTACCGTTAA & wbpK & gene & 3529508 & 3530458 & - \\
\hline Pseudomonas aeruginosa PAO1 & 3542936 & 3542945 & - & TTANCGTNAA & TTACCGTCAA & wbpB & gene & 3542740 & 3543690 & - \\
\hline Pseudomonas aeruginosa PAO1 & 3610559 & 3610568 & - & TTANCGTNAA & TTACCGTGAA & PA3222 & gene & 3610250 & 3611149 & + \\
\hline Pseudomonas aeruginosa PAO1 & 4075157 & 4075166 & - & TTANCGTNAA & TTATCGTTAA & dnaE & gene & 4075157 & 4078678 & - \\
\hline Pseudomonas aeruginosa PAO1 & 4626505 & 4626514 & + & TTANCGTNAA & TTACCGTCAA & & intergenic & & & \\
\hline Pseudomonas aeruginosa PAO1 & 4925851 & 4925860 & - & TTANCGTNAA & TTACCGTCAA & & intergenic & & & \\
\hline Pseudomonas aeruginosa PAO1 & 4974135 & 4974144 & + & TTANCGTNAA & TTAACGTAAA & & intergenic & & & \\
\hline Pseudomonas aeruginosa PAO1 & 5131447 & 5131456 & + & TTANCGTNAA & TTATCGTGAA & PA4582 & gene & 5131428 & 5132573 & + \\
\hline Pseudomonas aeruginosa PAO1 & 5242860 & 5242869 & + & TTANCGTNAA & TTAACGTTAA & & intergenic & & & \\
\hline
\end{tabular}




\begin{tabular}{|c|c|c|c|c|c|c|c|c|c|c|}
\hline Pseudomonas aeruginosa PAO1 & 5242860 & 5242869 & - & TTANCGTNAA & TTAACGTTAA & & intergenic & & & \\
\hline Pseudomonas aeruginosa $\mathrm{PAO} 1$ & 5923502 & 5923511 & - & TTANCGTNAA & TTATCGTGAA & fims & gene & 5923295 & 5924371 & - \\
\hline Pseudomonas aeruginosa PAO1 & 123740 & 123749 & + & TTANCGTTNA & TTAGCGTTGA & & intergenic & & & \\
\hline Pseudomonas aeruginosa $\mathrm{PAO1}$ & 1069111 & 1069120 & - & TTANCGTTNA & TTACCGTTGA & PA0987 & gene & 1068489 & 1069331 & + \\
\hline Pseudomonas aeruginosa PAO1 & 1087474 & 1087483 & + & TTANCGTTNA & TTAACGTTAA & & intergenic & & & \\
\hline Pseudomonas aeruginosa PAO1 & 1087474 & 1087483 & - & TTANCGTTNA & TTAACGTTAA & & intergenic & & & \\
\hline Pseudomonas aeruginosa PAO1 & 1467867 & 1467876 & + & TTANCGTTNA & TTACCGTTGA & PA1353 & gene & 1467488 & 1467901 & - \\
\hline Pseudomonas aeruginosa $\mathrm{PAO1}$ & 3120854 & 3120863 & - & TTANCGTTNA & TTACCGTTGA & oprQ & gene & 3120073 & 3121350 & + \\
\hline Pseudomonas aeruginosa PAO1 & 3530112 & 3530121 & + & TTANCGTTNA & TTACCGTTAA & wbpK & gene & 3529508 & 3530458 & - \\
\hline Pseudomonas aeruginosa $\mathrm{PAO1}$ & 4075157 & 4075166 & - & TTANCGTTNA & TTATCGTTAA & dnaE & gene & 4075157 & 4078678 & - \\
\hline Pseudomonas aeruginosa $\mathrm{PAO} 1$ & 5120078 & 5120087 & - & TTANCGTTNA & TTACCGTTCA & PA4571 & gene & 5118538 & 5120565 & + \\
\hline Pseudomonas aeruginosa PAO1 & 5242860 & 5242869 & - & TTANCGTTNA & TTAACGTTAA & & intergenic & & & \\
\hline Pseudomonas aeruginosa PAO1 & 5242860 & 5242869 & + & TTANCGTTNA & TTAACGTTAA & & intergenic & & & \\
\hline Pseudomonas aeruginosa PAO1 & 5680727 & 5680736 & + & TTANCGTTNA & TTAGCGTTCA & & intergenic & & & \\
\hline Pseudomonas aeruginosa PAO1 & 225242 & 225251 & - & TTANCGTTAN & TTATCGTTAT & & intergenic & & & \\
\hline Pseudomonas aeruginosa PAO1 & 1087474 & 1087483 & + & TTANCGTTAN & TTAACGTTAA & & intergenic & & & \\
\hline Pseudomonas aeruginosa PAO1 & 1087474 & 1087483 & - & TTANCGTTAN & TTAACGTTAA & & intergenic & & & \\
\hline Pseudomonas aeruginosa PAO1 & 1117641 & 1117650 & - & TTANCGTTAN & TTATCGTTAT & & intergenic & & & \\
\hline Pseudomonas aeruginosa PAO1 & 1665090 & 1665099 & + & TTANCGTTAN & TTATCGTTAT & zipA & gene & 1665065 & 1665934 & \\
\hline Pseudomonas aeruginosa PAO1 & 1666090 & 1666099 & + & TTANCGTTAN & TTACCGTTAC & lig & gene & 1666025 & 1668409 & \\
\hline Pseudomonas aeruginosa PAO1 & 1723297 & 1723306 & + & TTANCGTTAN & TTATCGTTAC & $\operatorname{sdhB}$ & gene & 1723280 & 1723987 & \\
\hline Pseudomonas aeruginosa PAO1 & 2544919 & 2544928 & - & TTANCGTTAN & TTACCGTTAC & ambB & gene & 2541197 & 2544946 & \\
\hline Pseudomonas aeruginosa PAO1 & 2556300 & 2556309 & + & TTANCGTTAN & TTACCGTTAT & PA2318 & gene & 2556293 & 2556658 & \\
\hline Pseudomonas aeruginosa PAO1 & 3483666 & 3483675 & - & TTANCGTTAN & TTATCGTTAT & хсрP & gene & 3483642 & 3484349 & \\
\hline Pseudomonas aeruginosa PAO1 & 3530112 & 3530121 & + & TTANCGTTAN & TTACCGTTAA & wbpK & gene & 3529508 & 3530458 & \\
\hline Pseudomonas aeruginosa $\mathrm{PAO} 1$ & 3683919 & 3683928 & + & TTANCGTTAN & TTATCGTTAT & tle 1 & gene & 3681500 & 3684148 & \\
\hline Pseudomonas aeruginosa PAO1 & 3893261 & 3893270 & - & TTANCGTTAN & TTACCGTTAG & & intergenic & & & \\
\hline Pseudomonas aeruginosa $\mathrm{PAO} 1$ & 4033928 & 4033937 & - & TTANCGTTAN & TTACCGTTAC & PA3598 & gene & 4033850 & 4034665 & \\
\hline Pseudomonas aeruginosa PAO1 & 4075157 & 4075166 & - & TTANCGTTAN & TTATCGTTAA & dnaE & gene & 4075157 & 4078678 & \\
\hline Pseudomonas aeruginosa PAO1 & 4609488 & 4609497 & - & TTANCGTTAN & TTAACGTTAT & & intergenic & & & \\
\hline Pseudomonas aeruginosa PAO1 & 4663708 & 4663717 & - & TTANCGTTAN & TTATCGTTAT & & intergenic & & & \\
\hline Pseudomonas aeruginosa PAO1 & 5242860 & 5242869 & + & TTANCGTTAN & TTAACGTTAA & & intergenic & & & \\
\hline Pseudomonas aeruginosa PAO1 & 5242860 & 5242869 & - & TTANCGTTAN & TTAACGTTAA & & intergenic & & & \\
\hline Pseudomonas aeruginosa PAO1 & 5786723 & 5786732 & + & TTANCGTTAN & TTATCGTTAT & & intergenic & & & \\
\hline
\end{tabular}

Table S2. Primers used in the work.

\begin{tabular}{|l|l|}
\hline Purpose/Name & Sequence (5'-3') \\
\hline Protein Expression & CTTTAAGAAGGAGATATACATATGATGGCTACCAATGGTATGC \\
\hline pET22b-hig $\boldsymbol{A}$-f & GTGGTGGTGGTGCTCGAGTCCGTGAGCAAGCAGCGGCTCAATC \\
\hline pET22b-hig $\boldsymbol{A}$-r & GCGACAGTGAACGATATCG \\
\hline pET22b-hig $\boldsymbol{A}$-P39A-f &
\end{tabular}




\begin{tabular}{|c|c|}
\hline pET22b-higA-P39A-r & AGCGGAGACTTTCAAAGC \\
\hline pME6032-higA-f & GCTAGTCCGAGGCCTCGAGATCATGGCTACCAATGGTATG \\
\hline pME6032-higA-r & CACACAGGAAACAGAATTCGAGCTATCCGTGAGCAAGCAG \\
\hline \multicolumn{2}{|l|}{ Gene knockout } \\
\hline pEX18-higA-upstream-f & AACGACGGCCAGTGCCAAGCTTCGGTGTTGAAGCGAGTGTC \\
\hline pEX18-higA-upstream-r & TAACAGTCTAGCTAGCGGTCCACCTCCTCAGTGGTA \\
\hline pEX18-higA-downstream-f & TAACAGTCTAGCTAGCCCGTAAATCGGTAAAGGG \\
\hline pEX18-higA-downstream-r & TTCGAGCTCGGTACCCGGGGATTCGCTGCTGAAGAACCTC \\
\hline pEX18-remove-higA-f & GGATTACACGTTGAAGCG \\
\hline pEX18-remove-higA-r & GAAAAGCGCATCGCCGATTCC \\
\hline pME6032-higA-f & GCTAGTCCGAGGCCTCGAGATCATGGCTACCAATGGTATG \\
\hline pME6032-higA-r & CACACAGGAAACAGAATTCGAGCTATCCGTGAGCAAGCAG \\
\hline \multicolumn{2}{|l|}{ qRT-PCR } \\
\hline $\operatorname{hig} A-\mathbf{f}$ & GTATGCGCCCCATCCATCCT \\
\hline $\operatorname{hig} A-\mathbf{r}$ & CAAAGTAACGCCCCAGACGA \\
\hline $\operatorname{hig} B-\mathbf{f}$ & TCTGACCTTTCGCTGCGACGAG \\
\hline $\operatorname{hig} B-\mathbf{r}$ & TCCCTGCAACGGCTCCAACC \\
\hline $\operatorname{exs} A-f$ & CTGGCGAGTTGCTTTTCGTC \\
\hline $\operatorname{exs} A-\mathbf{r}$ & ACGCTCGACTTCACTCAACA \\
\hline exoT-f & ACTGCATGCGGTAATGGACA \\
\hline exoT-r & CGCCGTTGGTATAGAGACCG \\
\hline $\mathbf{P}_{\text {exs } A^{-}} \mathbf{f}$ & CGCGACTGTTTTCCGAATTGT \\
\hline $\mathbf{P}_{\text {exs } A}-\mathbf{r}$ & AAGAACCCCAACACTTCCCG \\
\hline$a m r Z-f$ & AGCATGAACTCCGAGATC \\
\hline amrZ-r & AACACCGAGATTGTCTTG \\
\hline tss $J 1-f$ & AGAAACTGATCGTCGCGCTG \\
\hline tssJI-r & GACGGGTTGAGGTTCGGTG \\
\hline fha1-f & GGGGTGTACTACCTCACCGA \\
\hline fha1-r & GCTGGACGAGGATGTCGTAT \\
\hline tss $A 1-\mathrm{f}$ & CGATCGCCTGCTCGAGTATT \\
\hline tssA1-r & CGGGGATGAGATTCCTCACG \\
\hline hcpl-f & GGGTGAGTCCAAGGACAAGA \\
\hline hcp1-r & GGACACCAGGACTTCCTTCA \\
\hline $\operatorname{clpV1-f}$ & AACTGGGGCGGATCAAGAAG \\
\hline clpVI-r & AACAGCGTGTTGGTGAGGAT \\
\hline $\operatorname{vgrG1-\mathrm {f}}$ & ATGAAGAGCCGTTCGAGCAA \\
\hline vgrG1-r & CGCTCGGCGTGGATGTATAA \\
\hline oprL-f & TGCGATCACCACCTTCTACTTC \\
\hline oprL-r & CGCTGACCGCTGCCTTTC \\
\hline \multicolumn{2}{|l|}{ Reporter Plasmid } \\
\hline pRG970-exs $A$-f & GACTGACCTACCCGGGGATCCCCTTCGATCTGGAGGTCGACAGG \\
\hline pRG970-exs $A$-r & CTCTAGAAGAAGCTTGGGATCCATTATAAGAACCCCAACACTTCC \\
\hline pRG970-higB-f & GACTGACCTACCCGGGGATCCGATGGGCGGCAAAGCTAAAC \\
\hline
\end{tabular}




\begin{tabular}{|c|c|}
\hline pRG970-higB-r & CTCTAGAAGAAGCTTGGGATCCTCATTAACCCTTAACGTTAAGCG \\
\hline pRG970-amrZ-f & GACTGACCTACCCGGGGATCCAAAACTAGGCTTTTGCTG \\
\hline pRG970-amrZ-r & CTCTAGAAGAAGCTTGGGATCCACATTGAACCTGTAGAGTC \\
\hline pRG970-seqencing-F & ATTCAGGCTGCGCAACTG \\
\hline \multicolumn{2}{|l|}{ EMSA } \\
\hline $\operatorname{exs} A-\mathrm{f}$ & GAGATTAAAGAAGTGCAAGTCTCGTTCGTT \\
\hline $\operatorname{exs} A-\mathrm{r}$ & AACGAACGAGACTTGCACTTCTTTAATCTC \\
\hline Mutant-exs $A$-f & GAGATTAAAGAAGTGGGCAAGTCTCGTTCGTT \\
\hline Mutant-exs $A-\mathrm{r}$ & AACGAACGAGACTTGCCCACTTCTTTAATCTC \\
\hline$a m r Z-f$ & GCCGGCACGCAACGCACAATTGACGTCAACTAATTGTTGTTTCC \\
\hline$a m r Z-r$ & GGAAACAACAATTAGTTGACGTCAATTGTGCGTTGCGTGCCGGC \\
\hline Mutant-amrZ-f & GCCGGCACGCAACGCACAATTGACGGGTCAACTAATTGTTGTTTCC \\
\hline Mutant-amrZ-r & GGAAACAACAATTAGTTGACCCGTCAATTGTGCGTTGCGTGCCGGC \\
\hline
\end{tabular}

